\title{
Stationary Distributions of Second Order Stochastic Evolution Equations with Memory in Hilbert Spaces
}

\author{
Kai Liu \\ Department of Mathematical Sciences, \\ School of Physical Sciences, \\ The University of Liverpool, \\ Liverpool, L69 7ZL, U.K. \\ E-mail:k.liu@liverpool.ac.uk
}

\begin{abstract}
In this paper, we consider stationarity of a class of second-order stochastic evolution equations with memory, driven by Wiener processes or Lévy jump processes, in Hilbert spaces. The strategy is to formulate by reduction some first-order systems in connection with the stochastic equations under investigation. We develop asymptotic behavior of dissipative second-order equations and then apply them to time delay systems through Gearhart-PrüssGreiner's theorem. The stationary distribution of the system under consideration is the projection on the first coordinate of the corresponding stationary results of a lift-up stochastic system without delay on some product Hilbert space. Last, an example of stochastic damped delay wave equations with memory is presented to illustrate our theory.
\end{abstract}

Keywords: Stationary solutions; Second order stochastic evolution equations; Hereditary term.

2000 Mathematics Subject Classification(s): 60H15, 60G15, 60H05. 


\section{Introduction}

For any Hilbert spaces $H$ and $K$, we denote by $\mathscr{L}(H, K)$ the space of all bounded linear operators from $H$ into $K$. If $H=K$, we simply write $\mathscr{L}(H)$ for $\mathscr{L}(H, H)$. Let $r>0$ and consider the following second-order stochastic abstract Cauchy problem with memory on a Hilbert space $H$,

$$
\left\{\begin{array}{l}
d\left(\frac{d u(t)}{d t}\right)+A u(t) d t=B u^{\prime}(t) d t+M u_{t} d t+N u_{t}^{\prime} d t+R\left(u(t), u^{\prime}(t), u_{t}, u_{t}^{\prime}\right) d Z(t), \quad t \geq 0, \\
u(0)=\phi_{0,1}, \quad u^{\prime}(0)=\phi_{0,2}, \quad u_{0}=\phi_{1,1}, \quad u_{0}^{\prime}=\phi_{1,2},
\end{array}\right.
$$

where $\phi_{i, j}, i=0,1, j=1,2$, are appropriate initial data and $Z$ could be an abstract $Q$ Wiener process or Lévy jump process. Here $u_{t}(\theta):=u(t+\theta), u_{t}^{\prime}(\theta):=u^{\prime}(t+\theta), \theta \in[-r, 0]$ and $(A, \mathscr{D}(A)),(B, \mathscr{D}(B))$ are two linear operators from $H$ into itself, $M, N$ are linear mappings from $L^{2}([-r, 0], H)$ into $H$, respectively, and $R$ is a measurable nonlinear mapping from $H \times H \times L^{2}([-r, 0], H) \times L^{2}([-r, 0], H)$ to some space of linear operators (see Sections 4, 5 and 6 for precise definitions). In this work, we intend to consider stationarity of solutions for a class of stochastic second-order evolution equations with memory, i.e., (1.1).

We remark that there exists an exhaustive literature on deterministic second-order abstract Cauchy problems, e.g., see Fattorini [6] and references therein. There are some works devoted to this type of equations with memory features, and we refer, for instance, to [5]

for some fundamental statements and to [2] for some recent results about this topic. On the other hand, it is worth pointing out that stochastic abstract second-order Cauchy problems with memory such as (1.1) have not been investigated in depth until now. The only works known to the current author in the existing literature are [7, 8, 14, 15] in which the wellposedness and stability of stochastic systems with an infinite delay driven by a jump process and a neutral or impulsive term are considered.

The whole organisation of this work is as follows. After having developed some necessary tools to study the well-posedness of dissipative linear wave equations in Section 2, we shall consider the asymptotic behavior of abstract deterministic second-order evolution equations in Section 3 and some generalized second-order evolutions with memory in Section 4. We shall show in Section 5 how to reduce a second-order stochastic equation with memory to an abstract stochastic Cauchy problem on appropriate product Hilbert spaces. Consequently, we can use in Section 5 some recent methods to investigate the stationarity of the stochastic systems under investigation. In Section 6, we carry on to consider the stationarity of stochastic delay systems driven by a Lévy jump process. Last, we apply our theory to an illustrative example, i.e., a stochastic damped wave equation with memory in Section 7.

\section{Dissipative Wave Equations}

Consider the following second-order abstract Cauchy problem on a Hilbert space $H$,

$$
\left\{\begin{array}{l}
u^{\prime \prime}(t)+A u(t)=B u^{\prime}(t), \quad t \geq 0 \\
u(0)=\phi_{0,1}, \quad u^{\prime}(0)=\phi_{0,2}
\end{array}\right.
$$


where $B: \mathscr{D}(B) \subset H \rightarrow H$ is some linear, possibly unbounded, operator and $A: \mathscr{D}(A) \subset$ $H \rightarrow H$ is a self-adjoint linear operator such that

$$
\langle A u, u\rangle_{H} \geq \alpha\|u\|_{V}^{2}, \quad \alpha>0, \quad \forall x \in \mathscr{D}(A)
$$

where $V:=\mathscr{D}\left(A^{1 / 2}\right)$, equipped with the usual graph norm $\|u\|_{V}:=\left\|A^{1 / 2} u\right\|_{H}, u \in V$. By using the standard reduction $y(t):=\left(\begin{array}{c}u(t) \\ u^{\prime}(t)\end{array}\right)$, we intend to transform (2.1) into a first-order system

$$
\left\{\begin{array}{l}
y^{\prime}(t)=\Lambda y(t), \quad t \geq 0 \\
y(0)=\left(\begin{array}{l}
\phi_{0,1} \\
\phi_{0,2}
\end{array}\right) \in \mathbb{H}
\end{array}\right.
$$

for the matrix operator

$$
\Lambda=\left(\begin{array}{cc}
0 & I \\
-A & B
\end{array}\right)
$$

with domain

$$
\mathscr{D}(\Lambda)=\mathscr{D}(A) \times\left(\mathscr{D}\left(A^{1 / 2}\right) \cap \mathscr{D}(B)\right)
$$

in the product Hilbert space $\mathbb{H}:=V \times H$, equipped with the usual product space inner product and norm. To this end, we remark that $A^{1 / 2}: V \rightarrow H$ is a unitary operator. This allows us to consider a unitary operator

$$
\Sigma=\left(\begin{array}{cc}
A^{1 / 2} & 0 \\
0 & I
\end{array}\right) \in \mathscr{L}(\mathbb{H}, \tilde{\mathbb{H}}),
$$

where $I$ is the identity operator on $H$ and $\tilde{\mathbb{H}}:=H \times H$, equipped with the usual product space inner product and norm. Further, define a matrix operator on $\tilde{\mathbb{H}}$,

$$
\Lambda_{0}=\left(\begin{array}{cc}
0 & A^{1 / 2} \\
-A^{1 / 2} & B
\end{array}\right)
$$

with domain

$$
\mathscr{D}\left(\Lambda_{0}\right)=\mathscr{D}\left(A^{1 / 2}\right) \times\left(\mathscr{D}\left(A^{1 / 2}\right) \cap \mathscr{D}(B)\right) .
$$

Then we know that $\Sigma$ defines a unitary equivalence between $\Lambda$ and $\Lambda_{0}$, i.e.,

$$
\Lambda_{0}=\Sigma \Lambda \Sigma^{-1}
$$

Hence, in order to deal with the well-posedness of (2.2) it suffices to consider the same problem of the following equation

$$
\left\{\begin{array}{l}
y^{\prime}(t)=\Lambda_{0} y(t), \quad t \geq 0 \\
y(0)=\left(\begin{array}{l}
\phi_{0,1} \\
\phi_{0,2}
\end{array}\right) \in \tilde{\mathbb{H}}
\end{array}\right.
$$

in the product Hilbert space $\tilde{\mathbb{H}}$. 
To proceed further, we want to find conditions under which the inverse operator $\Lambda_{0}^{-1}$ of $\Lambda_{0}$ exists. In fact, if $\Lambda_{0}$ is invertible, we know by the closed graph theorem that $\Lambda_{0}^{-1}$ is a bounded linear operator on $\tilde{\mathbb{H}}$ and we can represent it in a matrix form

$$
\Lambda_{0}^{-1}=\left(\begin{array}{cc}
U & V \\
W & S
\end{array}\right) \in \mathscr{L}(\tilde{\mathbb{H}})
$$

To identify $\Lambda_{0}^{-1}$ explicitly, we can formally conclude from $\Lambda_{0}^{-1} \Lambda_{0}=\left.I\right|_{\mathscr{D}\left(\Lambda_{0}\right)}$ that its entries satisfy

$$
\begin{aligned}
& U x=A^{-1 / 2} B A^{-1 / 2} x \quad \text { for all } \quad x \in \mathscr{D}\left(A^{-1 / 2} B A^{-1 / 2}\right), \\
& V=-A^{-1 / 2}, W=A^{-1 / 2} \text { and } S=0 .
\end{aligned}
$$

These operators give rise to a bounded inverse of $\Lambda_{0}$ if and only if $A^{1 / 2}\left(\mathscr{D}(B) \cap \mathscr{D}\left(A^{1 / 2}\right)\right)$ is dense in $H$ and $A^{-1 / 2} B A^{-1 / 2} \in \mathscr{L}(H)$. As a matter of fact, we have the following result.

Theorem 2.1. Assume that $B: \mathscr{D}(B) \subset H \rightarrow H$ is densely defined, closed and dissipative, i.e., $\operatorname{Re}\langle B u, u\rangle_{H} \leq 0$ for all $u \in \mathscr{D}(B)$ and $\mathscr{D}(B) \cap \mathscr{D}\left(A^{1 / 2}\right)$ is dense in $H$. Then operator $\Lambda_{0}$ is densely defined, dissipative and closed. Further, suppose that $A^{1 / 2}\left(\mathscr{D}(B) \cap \mathscr{D}\left(A^{1 / 2}\right)\right)$ is dense in $H$ and $A^{-1 / 2} B A^{-1 / 2} \in \mathscr{L}(H)$, then $\Lambda_{0}$ generates a contraction $C_{0}$-semigroup $e^{t \Lambda_{0}}$, $t \geq 0$, on $\tilde{\mathbb{H}}$, i.e., $\left\|e^{t \Lambda_{0}}\right\| \leq 1$ for all $t \geq 0$.

Proof. It is immediate that $\Lambda_{0}$ is densely defined and closed. Now we show the dissipativity of $\Lambda_{0}$. By assumption, since $B$ is dissipative, we have for any $\left(\begin{array}{l}u \\ v\end{array}\right) \in \mathscr{D}\left(\Lambda_{0}\right)$ that

$$
\begin{aligned}
\operatorname{Re}\left\langle\Lambda_{0}\left(\begin{array}{l}
u \\
v
\end{array}\right),\left(\begin{array}{l}
u \\
v
\end{array}\right)\right\rangle_{\tilde{\mathbb{H}}} & =\operatorname{Re}\left\langle\left(\begin{array}{cc}
0 & A^{1 / 2} \\
-A^{1 / 2} & B
\end{array}\right)\left(\begin{array}{l}
u \\
v
\end{array}\right),\left(\begin{array}{l}
u \\
v
\end{array}\right)\right\rangle_{\tilde{\mathbb{H}}} \\
& =\operatorname{Re}\left\langle A^{1 / 2} v, u\right\rangle_{H}+\operatorname{Re}\left\langle-A^{1 / 2} u+B v, v\right\rangle_{H} \\
& =\operatorname{Re}\langle B v, v\rangle_{H} \leq 0,
\end{aligned}
$$

i.e., $\Lambda_{0}$ is dissipative.

On the other hand, by assumption, $\Lambda_{0}$ has a bounded linear inverse $\Lambda_{0}^{-1}, 0 \in \rho\left(\Lambda_{0}\right)$ which is an open set in $\mathbb{C}$. Hence, there exists a number $\lambda>0$ such that $\lambda \in \rho\left(\Lambda_{0}\right)$ and $\mathscr{R}\left(\lambda I-\Lambda_{0}\right)=\tilde{\mathbb{H}}$. By the well-known Lumer-Phillips Theorem, this implies that $\Lambda_{0}$ generates a contraction $C_{0}$-semigroup on $\tilde{\mathbb{H}}$.

Corollary 2.1. Assume that $B: \mathscr{D}(B) \subset H \rightarrow H$ is closed, dissipative and $\mathscr{D}\left(A^{1 / 2}\right) \subset$ $\mathscr{D}(B)$, then $\Lambda_{0}$ generates a contraction $C_{0}$-semigroup $e^{t \Lambda_{0}}, t \geq 0$, on $\tilde{\mathbb{H}}$.

Proof. The proof is straightaway since all the conditions in Theorem 2.1 are satisfied on this occasion.

In the sequel of this work, the conditions in Theorem 2.1 are always assumed to hold. 


\section{Asymptotic Behavior of Equations}

In this section, we are concerned with the asymptotic behavior of equation (2.1). We first establish a result for a simple case, i.e., $B=\beta I, \beta \in \mathbb{R}$, whose argument is shaped by J. Zabczyk and the current author, to show that the strict dissipativity of $B$ is essential for the exponential stability of (2.2), i.e., $\left\|e^{t \Lambda}\right\| \leq M e^{-\mu t}, t \geq 0$, for some constants $M \geq 1$ and $\mu>0$.

Suppose that $B=\beta I, \beta \in \mathbb{R}$, in (2.1). First, note that from the definition of the resolvent sets $\rho(-A)$ and $\rho(\Lambda)$, we have that $\lambda \in \rho(\Lambda)$ if and only if $\lambda(\lambda-\beta) \in \rho(-A)$. Indeed, $\lambda \in \rho(\Lambda)$ if and only if the following equation system defining resolvent operators

$$
\left(\begin{array}{cc}
\lambda I & -I \\
A & \lambda I-\beta I
\end{array}\right)\left(\begin{array}{l}
y_{1} \\
y_{2}
\end{array}\right)=\left(\begin{array}{l}
z_{1} \\
z_{2}
\end{array}\right)
$$

has a unique solution in $\mathscr{D}(\Lambda)$ for any $\left(z_{1}, z_{2}\right) \in \mathbb{H}$, a situation which is possible if and only if $\lambda(\lambda-\beta) \in \rho(-A)$. Let $\sigma(\Lambda)$ denote the spectral set of $\Lambda$ and define the spectral bound $\omega_{s}(\Lambda)$ of $\Lambda$ by

$$
\omega_{s}(\Lambda)=\sup \{\operatorname{Re} \lambda: \lambda \in \sigma(\Lambda)\}
$$

then we have

$$
\omega_{s}(\Lambda)=\sup \{\operatorname{Re} \lambda: \lambda(\lambda-\beta) \in \sigma(-A)\} .
$$

In other words, $\omega_{s}(\Lambda)$ is the larger real part of the roots to the equation

$$
\lambda^{2}-\beta \lambda-\omega_{s}(-A)=0
$$

with $\lambda(\lambda-\beta) \in \sigma(-A)$. Since $-A$ is self-adjoint, $\sigma(-A)$ is a subset of $\mathbb{R}$ and so we have $\lambda(\lambda-\beta) \in \mathbb{R}$. Let $\lambda=a+i b$. Since $(a+i b)(a+i b-\beta) \in \mathbb{R}$, it follows that

$$
b=0 \quad \text { or } \quad b \neq 0,2 a-\beta=0 .
$$

If $b=0$, this means that $\omega_{s}(\Lambda)$ equals the larger real root of (3.1), which is

$$
\omega_{s}(\Lambda)=\frac{\beta}{2}+\sqrt{\frac{\beta^{2}}{4}+\omega_{s}(-A)} \quad \text { if } \quad \frac{\beta^{2}}{4}+\omega_{s}(-A) \geq 0 .
$$

If $b \neq 0$ and $2 a-\beta=0$, this means that (3.1) has non real roots in this case and $\omega_{s}(\Lambda)=$ $a=\beta / 2$. In other words, we have the following relation of spectrum bounds between $\omega_{s}(-A)$ and $\omega_{s}(\Lambda)$

$$
\omega_{s}(\Lambda)= \begin{cases}\frac{\beta}{2}+\sqrt{\frac{\beta^{2}}{4}+\omega_{s}(-A)} & \text { if } \frac{\beta^{2}}{4}+\omega_{s}(-A) \geq 0, \\ \frac{\beta}{2} & \text { otherwise. }\end{cases}
$$

Define the growth bound $\omega_{g}(\Lambda)$ of $\Lambda$ by

$$
\omega_{g}(\Lambda)=\inf \left\{\mu \in \mathbb{R}: \text { there exists } M \geq 1 \text { such that }\left\|e^{t \Lambda}\right\| \leq M e^{\mu t} \text { for all } t \geq 0\right\}
$$


From (3.2), we have immediately the following conclusions (i) and (ii).

(i) If $\omega_{s}(-A) \geq 0$, we have $\omega_{g}(\Lambda) \geq \omega_{s}(\Lambda) \geq 0$ from (3.2) and system (2.2) is thus exponentially unstable for any $\beta \in \mathbb{R}$.

(ii) If $\omega_{s}(-A)<0$ and $\beta \geq 0$, then (3.2) implies that $\omega_{g}(\Lambda) \geq \omega_{s}(\Lambda) \geq 0$, and system (2.2) is thus exponentially unstable.

(iii) If $\omega_{s}(-A)<0$ and $\beta=-\alpha, \alpha>0, B=-\alpha I$, we may show that the operator

$$
P=\left(\begin{array}{cc}
\frac{1}{\alpha} I+\frac{\alpha}{2} A^{-1} & \frac{1}{2} A^{-1} \\
\frac{1}{2} I & \frac{1}{\alpha} I
\end{array}\right)
$$

is the unique self-adjoint, non-negative solution of Lyapunov equation

$$
\left\langle\Lambda\left(\begin{array}{l}
y_{1} \\
y_{2}
\end{array}\right), P\left(\begin{array}{l}
y_{1} \\
y_{2}
\end{array}\right)\right\rangle_{\mathbb{H}}+\left\langle P\left(\begin{array}{l}
y_{1} \\
y_{2}
\end{array}\right), \Lambda\left(\begin{array}{l}
y_{1} \\
y_{2}
\end{array}\right)\right\rangle_{\mathbb{H}}=-\left\|\left(\begin{array}{l}
y_{1} \\
y_{2}
\end{array}\right)\right\|_{\mathbb{H}}^{2}
$$

for any $y_{1} \in \mathscr{D}(A), y_{2} \in \mathscr{D}\left(A^{1 / 2}\right)$. In this case, the following estimates hold:

$$
\gamma_{-}\left\|\left(\begin{array}{l}
y_{1} \\
y_{2}
\end{array}\right)\right\|_{\mathbb{H}}^{2} \leq\left\langle P\left(\begin{array}{l}
y_{1} \\
y_{2}
\end{array}\right),\left(\begin{array}{l}
y_{1} \\
y_{2}
\end{array}\right)\right\rangle_{\mathbb{H}} \leq \gamma_{+}\left\|\left(\begin{array}{l}
y_{1} \\
y_{2}
\end{array}\right)\right\|_{\mathbb{H}}^{2}, \quad\left(\begin{array}{l}
y_{1} \\
y_{2}
\end{array}\right) \in \mathbb{H},
$$

where

$$
\gamma_{-}=\frac{1}{\alpha} \cdot \frac{\sqrt{1+\theta}}{1+\sqrt{1+\theta}}>0, \quad \gamma_{+}=\frac{1}{\alpha}\left(1+\frac{1+\sqrt{1+\theta}}{\theta}\right)>0, \quad \theta=\frac{4\left|\omega_{s}(-A)\right|}{\alpha^{2}} .
$$

Moreover, we have that

$$
\left\|e^{t \Lambda}\right\| \leq \sqrt{\frac{\gamma_{+}}{\gamma_{-}}} e^{-\frac{1}{2 \gamma_{+}} t}, \quad t \geq 0 .
$$

That is, system (2.2) in this case is exponentially stable.

Indeed, it is straightforward to get symmetry and nonnegativity of $P$ and (3.4) is easily verified by a direct calculation. To show (3.5), note that for any $\left(\begin{array}{l}y_{1} \\ y_{2}\end{array}\right) \in \mathbb{H}$,

$$
\left\langle P\left(\begin{array}{l}
y_{1} \\
y_{2}
\end{array}\right),\left(\begin{array}{l}
y_{1} \\
y_{2}
\end{array}\right)\right\rangle_{\mathbb{H}}=\frac{1}{\alpha}\left(\left\|A^{1 / 2} y_{1}\right\|_{H}^{2}+\frac{\alpha^{2}}{2}\left\|y_{1}\right\|_{H}^{2}+\alpha\left\langle y_{1}, y_{2}\right\rangle_{H}+\left\|y_{2}\right\|_{H}^{2}\right) .
$$

We first find the maximal $\gamma \geq 0$ such that for all $\left(\begin{array}{l}y_{1} \\ y_{2}\end{array}\right) \in \mathbb{H}$ :

$$
\frac{1}{\alpha}\left(\left\|A^{1 / 2} y_{1}\right\|_{H}^{2}+\frac{\alpha^{2}}{2}\left\|y_{1}\right\|_{H}^{2}+\alpha\left\langle y_{1}, y_{2}\right\rangle_{H}+\left\|y_{2}\right\|_{H}^{2}\right) \geq \gamma\left(\left\|A^{1 / 2} y_{1}\right\|_{H}^{2}+\left\|y_{2}\right\|_{H}^{2}\right) .
$$


If $\gamma=1 / \alpha$, inequality (3.7) becomes

$$
\frac{\alpha^{2}}{2}\left\|y_{1}\right\|_{H}^{2}+\alpha\left\langle y_{1}, y_{2}\right\rangle_{H} \geq 0
$$

which clearly does not hold for all $\left(\begin{array}{l}y_{1} \\ y_{2}\end{array}\right) \in \mathbb{H}$, e.g., let $y_{2}=-\alpha y_{1}, y_{1} \neq 0$. Therefore, $\gamma \in[0,1 / \alpha)$ and inequality (3.7) becomes

$$
(1-\alpha \gamma)\left\|A^{1 / 2} y_{1}\right\|_{H}^{2}+\frac{\alpha^{2}}{2}\left\|y_{1}\right\|_{H}^{2}+\alpha\left\langle y_{1}, y_{2}\right\rangle_{H}+(1-\alpha \gamma)\left\|y_{2}\right\|_{H}^{2} \geq 0
$$

On the other hand, for fixed $y_{1}$, we have

$$
\begin{aligned}
\min _{y_{2} \in H}\left\{\alpha\left\langle y_{1}, y_{2}\right\rangle_{H}+(1-\alpha \gamma)\left\|y_{2}\right\|_{H}^{2}\right\} & =\min _{y_{2} \in H}\left\{(1-\alpha \gamma)\left\|y_{2}+\frac{\alpha y_{1}}{2(1-\alpha \gamma)}\right\|_{H}^{2}-\frac{\alpha^{2}\left\|y_{1}\right\|_{H}^{2}}{4(1-\alpha \gamma)}\right\} \\
& =-\frac{\alpha^{2}}{4(1-\alpha \gamma)}\left\|y_{1}\right\|_{H}^{2} .
\end{aligned}
$$

Therefore, the required $\gamma \in[0,1 / \alpha)$ should be such that for $y_{1} \in \mathscr{D}\left(A^{1 / 2}\right)$,

$$
(1-\alpha \gamma)\left\|A^{1 / 2} y_{1}\right\|_{H}^{2} \geq \frac{\alpha^{2}}{2}\left(\frac{1}{2(1-\alpha \gamma)}-1\right)\left\|y_{1}\right\|_{H}^{2}
$$

Since $A$ is self-adjoint, it is well known that

$$
\left|\omega_{s}(-A)\right|=\inf _{y_{1} \neq 0} \frac{\left\|A^{1 / 2} y_{1}\right\|_{H}^{2}}{\left\|y_{1}\right\|_{H}^{2}} .
$$

This implies, in addition to (3.8), that one is equivalently looking for the maximum value $\gamma \in[0,1 / \alpha)$ such that

$$
\left|\omega_{s}(-A)\right| \geq \frac{\alpha^{2}}{4}\left(\frac{1}{(1-\alpha \gamma)^{2}}-\frac{2}{1-\alpha \gamma}\right),
$$

or,

$$
\theta \geq \frac{1}{(1-\alpha \gamma)^{2}}-\frac{2}{1-\alpha \gamma}
$$

This easily gives that

$$
\gamma_{-}=\frac{1}{\alpha} \cdot \frac{\sqrt{1+\theta}}{1+\sqrt{1+\theta}}
$$

In a similar way, the expression for $\gamma_{+}$can be obtained by looking for a minimum value $\gamma>0$ such that for all $\left(\begin{array}{l}y_{1} \\ y_{2}\end{array}\right) \in \mathbb{H}$ :

$$
\frac{1}{\alpha}\left(\left\|A^{1 / 2} y_{1}\right\|_{H}^{2}+\frac{\alpha^{2}}{2}\left\|y_{1}\right\|_{H}^{2}+\alpha\left\langle y_{1}, y_{2}\right\rangle_{H}+\left\|y_{1}\right\|_{H}^{2}\right) \leq \gamma\left(\left\|A^{1 / 2} y_{1}\right\|_{H}^{2}+\left\|y_{1}\right\|_{H}^{2}\right) .
$$


To prove the final part, we consider the mild solution $y$ of the problem (2.2). Then from Lyapunov equation (3.4), we have

$$
\frac{d}{d t}\langle P y(t), y(t)\rangle_{\mathbb{H}}=-\|y(t)\|_{\mathbb{H}}^{2}, \quad t \geq 0
$$

which, in addition to (3.5), immediately implies that

$$
\frac{d}{d t}\langle P y(t), y(t)\rangle_{\mathbb{H}}=-\|y(t)\|_{\mathbb{H}}^{2} \leq-\frac{1}{\gamma_{+}}\langle P y(t), y(t)\rangle_{\mathbb{H}}, \quad t \geq 0
$$

A simple calculation further yields that

$$
\langle P y(t), y(t)\rangle_{\mathbb{H}} \leq e^{-\frac{1}{\gamma_{+}} t}\langle P y(0), y(0)\rangle_{\mathbb{H}} \leq \gamma_{+} e^{-\frac{1}{\gamma_{+}} t}\|y(0)\|_{\mathbb{H}}^{2}, \quad t \geq 0
$$

Hence, we have

$$
\|y(t)\|_{\mathbb{H}}^{2} \leq \frac{1}{\gamma_{-}}\langle P y(t), y(t)\rangle_{\mathbb{H}} \leq \frac{\gamma_{+}}{\gamma_{-}} e^{-\frac{1}{\gamma_{+}} t}\|y(0)\|_{\mathbb{H}}^{2}, \quad t \geq 0
$$

as desired.

Next, we consider the case that $B$ is a linear unbounded operator. We first establish a useful lemma.

Lemma 3.1. Assume that there exist constants $\gamma \geq 0, \alpha>0$ such that

$$
\gamma \operatorname{Re}\langle B v, v\rangle_{H} \leq-\left|\operatorname{Im}\langle B v, v\rangle_{H}\right|, \quad \forall v \in \mathscr{D}(B)
$$

and

$$
R e\langle B v, v\rangle_{H} \leq-2 \alpha\|v\|_{H}^{2}, \quad \forall v \in \mathscr{D}(B) .
$$

Let $0<\delta<\alpha$ and $\delta-\alpha<a \leq 0$. If the inequality

$$
\inf _{y \in \mathscr{D}(\Lambda),\|y\|_{\mathbb{H}}=1}\|(a+i b) y-\Lambda y\|_{\mathbb{H}}<\delta, \quad b \in \mathbb{R}
$$

holds, then

$$
|b|<\alpha \cdot \frac{3 \delta+(\delta-a) \gamma}{\alpha-\delta+a}
$$

Proof. Let $y=\left(\begin{array}{l}u \\ v\end{array}\right) \in \mathscr{D}(\Lambda)$ with $\|y\|_{\mathbb{H}}^{2}=\left\|A^{1 / 2} u\right\|_{H}^{2}+\|v\|_{H}^{2}=1$ and for $a \in(\delta-\alpha, 0]$, $b \in \mathbb{R}$,

$$
\|(a+i b) y-\Lambda y\|_{\mathbb{H}}=\left\|\left(\begin{array}{c}
a u+i b u-v \\
A u+a v+i b v-B v
\end{array}\right)\right\|_{\mathbb{H}}<\delta .
$$

From (3.11), it easily follows that

$$
\left|\langle(a+i b) y-\Lambda y, y\rangle_{\mathbb{H}}\right| \leq\|((a+i b) I-\Lambda) y\|_{\mathbb{H}} \cdot\|y\|_{\mathbb{H}}<\delta,
$$


which yields

$$
\left|\langle(a+i b) y, y\rangle_{\mathbb{H}}+2 i \operatorname{Im}\langle u, A v\rangle_{H}-\langle B v, v\rangle_{H}\right|<\delta,
$$

and further, by considering the real parts in (3.12),

$$
\left|R e\langle B v, v\rangle_{H}-a\right|<\delta .
$$

Since $\left\|A^{1 / 2} u\right\|_{H}^{2}+\|v\|_{H}^{2}=1$ and $a>\delta-\alpha$, we have by virtue of (3.10) and (3.13) that

$$
\left|1-2\left\|A^{1 / 2} u\right\|_{H}^{2}\right|=\left|1-2+2\|v\|_{H}^{2}\right| \geq \frac{\operatorname{Re}\langle B v, v\rangle_{H}}{\alpha}+1>1-\frac{\delta-a}{\alpha} .
$$

On the other hand, we obtain from (3.11) the estimate

$$
\left|\left\langle a A^{1 / 2} u+i b A^{1 / 2} u-A^{1 / 2} v, A^{1 / 2} u\right\rangle_{H}\right| \leq\left\|a A^{1 / 2} u+i b A^{1 / 2} u-A^{1 / 2} v\right\|_{H} \cdot\left\|A^{1 / 2} u\right\|_{H}<\delta,
$$

hence, by taking imaginary parts,

$$
\left|\operatorname{Im}\langle u, A v\rangle_{H}+b\left\|A^{1 / 2} u\right\|_{H}^{2}\right|<\delta .
$$

Last, by considering the imaginary part of (3.12), we have

$$
\left|b+2 \operatorname{Im}\langle u, A v\rangle_{H}-\operatorname{Im}\langle B v, v\rangle_{H}\right|<\delta,
$$

which, in addition to (3.16), yields

$$
\begin{aligned}
|b| \cdot\left|1-2\left\|A^{1 / 2} u\right\|_{H}^{2}\right| & -\left|\operatorname{Im}\langle B v, v\rangle_{H}\right| \\
& \leq\left|b\left(1-2\left\|A^{1 / 2} u\right\|_{H}^{2}\right)-\operatorname{Im}\langle B v, v\rangle_{H}\right| \\
& \leq\left|2 \operatorname{Im}\langle u, A v\rangle_{H}+2 b\left\|A^{1 / 2} u\right\|_{H}^{2}\right|+\left|b+2 \operatorname{Im}\langle u, A v\rangle_{H}-\operatorname{Im}\langle B v, v\rangle_{H}\right| \\
& \leq 3 \delta .
\end{aligned}
$$

This relation implies, together with (3.9), (3.13) and (3.14), that

$$
|b|\left(1-\frac{\delta-a}{\alpha}\right) \leq 3 \delta-\gamma \operatorname{Re}\langle B v, v\rangle_{H}<3 \delta+(\delta-a) \gamma,
$$

and the assertion follows.

To proceed further, we need the powerful Gearhart-Prüss-Greiner's lemma whose proof is referred to Theorem 1.11 and Exercise 1.13, pp. 302-304, in [5]. For any linear operator $A$, let $R(\lambda, A)$ denote the resolvent operator of $A, \lambda \in \rho(A)$.

Lemma 3.2. (Gearhart-Prüss-Greiner) For a strongly continuous semigroup $e^{t A}, t \geq 0$, with generator $A$ on a Hilbert space $H$, its growth bound $\omega_{g}(A)$ is given by

$$
\omega_{g}(A)=\inf \left\{a>\omega_{s}(A): \sup _{b \in \mathbb{R}}\|R(a+i b, A)\|<\infty\right\},
$$

where $\omega_{s}(A)$ is the spectral bound of $A$. In particular, $e^{t A}, t \geq 0$, is exponentially stable if and only if the half-plane $\{\lambda=a+i b \in \mathbb{C}: a>0, b \in \mathbb{R}\}$ is contained in the resolvent set $\rho(A)$ of $A$ with the resolvent satisfying

$$
\sup _{a>0, b \in \mathbb{R}}\|R(a+i b, A)\|<\infty .
$$


Proposition 3.1. Assume that (3.9) and (3.10) hold. Let $\Lambda$ be the operator in (2.2) which generates a contraction $C_{0}$-semigroup $e^{t \Lambda}, t \geq 0$, on $\mathbb{H}$. Then the growth bound $w_{g}(\Lambda)$ of $\Lambda$ satisfies

$$
w_{g}(\Lambda) \leq \max \left\{w_{s}(\Lambda),-\alpha\right\}<0
$$

Proof. First note that since $\Lambda$ generates a contraction semigroup, it is immediate that

$$
\{\lambda \in \mathbb{C}: \operatorname{Re} \lambda>0\} \subset \rho(\Lambda),
$$

i.e., $w_{s}(\Lambda) \leq 0$.

To show $w_{s}(\Lambda)<0$, we first verify by contraction that $\sigma(\Lambda) \cap i \mathbb{R}=\emptyset$. Assume that there exists $b_{0} \in \mathbb{R}$ such that $i b_{0} \in \sigma(\Lambda)$. If $b_{0}=0$, then $0 \in \sigma(\Lambda)$, a fact which contradicts the assumption $\Lambda^{-1} \in \mathscr{L}(\mathbb{H})$. On the other hand, if $b_{0} \neq 0$, it is always possible to find a number $\delta>0$ small enough such that $\left|b_{0}\right| \geq \alpha \frac{3 \delta+\delta \gamma}{\alpha-\delta}$ in Lemma 3.1, and so

$$
\inf _{y \in \mathscr{D}(\Lambda),\|y\|_{\mathbb{H}}=1}\left\|i b_{0} y-\Lambda y\right\|_{\mathbb{H}} \geq \delta
$$

which, by definition, implies that $i b_{0} \notin \sigma_{a p}(\Lambda)$. Here $\sigma_{a p}(\Lambda)$ is the approximate point spectrum set of $\Lambda$, defined by

$$
\sigma_{a p}(\Lambda)=\{\lambda \in \mathbb{C}: \lambda I-\Lambda \text { is not injective or } \mathscr{R}(\lambda I-\Lambda) \text { is not closed in } \mathbb{H}\},
$$

where $\mathscr{R}(\lambda I-\Lambda)$ is the range of operator $\lambda I-\Lambda$. But this is a contradiction, since $i b_{0} \in \partial \sigma(\Lambda)$ which is a subset of $\sigma_{a p}(\Lambda)$ (cf. Proposition 1.10, pp. 242-243, in [5]). Hence, $i \mathbb{R} \in \rho(\Lambda)$. Now, let $a=0$ and $|b| \geq \alpha \cdot \frac{3 \delta+\delta \gamma}{\alpha-\delta}$ for $0<\delta<\alpha$ in Lemma 3.1, it follows immediately that

$$
\inf _{y \in \mathscr{D}(\Lambda),\|y\|_{\mathbb{H}}=1}\|i b y-\Lambda y\|_{\mathbb{H}} \geq \delta, \quad \text { or equivalently, } \quad\|R(i b, \Lambda)\| \leq \frac{1}{\delta} .
$$

On the other hand, $\|R(\lambda, \Lambda)\|, \lambda \in \rho(\Lambda)$, is bounded on any compact set of $i \mathbb{R}$. Hence, $w_{s}(\Lambda)<0$.

Last, according to Gearhart-Prüss-Greiner's lemma, we want to show

$$
a+i \mathbb{R} \subset \rho(\Lambda) \quad \text { and } \quad \sup _{b \in \mathbb{R}}\|R(a+i b, \Lambda)\|<\infty \quad \text { for all } a>\max \left\{w_{s}(\Lambda),-\alpha\right\} .
$$

Indeed, if $a>\max \left\{w_{s}(\Lambda),-\alpha\right\}$ and

$$
|b| \geq \alpha \cdot \frac{3 \delta+(\delta-a) \gamma}{\alpha-\delta+a} \quad \text { for } \quad 0<\delta<\alpha
$$

in Lemma 3.1, then $a+i \mathbb{R} \subset \rho(\Lambda)$ and

$$
\sup _{\left\{b:|b| \geq \alpha \frac{3 \delta+(\delta-a) \gamma}{\alpha-\delta+a}\right\}}\|R(a+i b, \Lambda)\| \leq \frac{1}{\delta} .
$$

On the other hand, $\|R(\lambda, \Lambda)\|, \lambda \in \rho(\Lambda)$, is bounded on any compact set of $\mathbb{C}$. Hence, the desired (3.17) holds. The proof is complete now. 
Corollary 3.1. Under the same conditions as in Proposition 3.1,

(i) if $\gamma \neq 0$, then

$$
w_{g}(\Lambda) \leq \nu
$$

where $\nu \in(-\alpha, 0)$ is the unique solution of the equation

$$
\nu^{2}+\left(\frac{\nu \gamma \alpha}{\alpha+\nu}\right)^{2}=\left\|\Lambda^{-1}\right\|^{-2}
$$

(ii) if $\gamma=0$, then

$$
w_{g}(\Lambda) \leq \max \left\{-\alpha,-\left\|\Lambda^{-1}\right\|^{-1}\right\} .
$$

Proof. For $\gamma \neq 0$, if $a \in(-\alpha, 0]$ and $|b|>\frac{-\alpha \gamma a}{a+\alpha}$, then there exists a sufficiently small $\delta>0$ such that $-\alpha+\delta<a \leq 0$ and

$$
|b| \geq \alpha \cdot \frac{3 \delta+(\delta-a) \gamma}{\alpha-\delta+a} .
$$

By virtue of Lemma 3.1, it thus implies that $a+i b \in \rho(\Lambda)$. That is, the curve

$$
\left(\nu, \frac{\nu \gamma \alpha}{\alpha+\nu}\right), \quad \nu \in(-\alpha, 0)
$$

is contained in the resolvent set. On the other hand, it is easy to know that the disk with radius $\left\|\Lambda^{-1}\right\|^{-1}$ centered at zero is contained in the resolvent set (cf. Theorem 2.3, p. 274 in [16]). We thus obtain the desired (i) by intersecting the two curves. For (ii), we choose first $\gamma>0$, use (i) and then take the limit as $\gamma \rightarrow 0$.

Corollary 3.2. Under the same conditions as in Proposition 3.1,

(i) if $\gamma \neq 0$, then

$$
w_{g}(\Lambda) \leq \nu,
$$

where $\nu \in(-\alpha, 0)$ is the unique solution of the equation

$$
\nu^{2}+\left(\frac{\nu \gamma \alpha}{\alpha+\nu}\right)^{2}=\left(\left\|A^{-1 / 2} B A^{-1 / 2}\right\|+2\left\|A^{-1 / 2}\right\|\right)^{-2} ;
$$

(ii) if $\gamma=0$, then

$$
w_{g}(\Lambda) \leq \max \left\{-\alpha,-\left(\left\|A^{-1 / 2} B A^{-1 / 2}\right\|+2\left\|A^{-1 / 2}\right\|\right)^{-1}\right\} .
$$

Proof. For any $\left(\begin{array}{l}u \\ v\end{array}\right) \in \mathscr{D}\left(\Lambda_{0}\right)$, we have

$$
\begin{aligned}
\left\|\Lambda_{0}^{-1}\left(\begin{array}{l}
u \\
v
\end{array}\right)\right\|_{\tilde{\mathbb{H}}} & =\left(\left\|A^{-1 / 2} B A^{-1 / 2} u-A^{-1 / 2} v\right\|_{H}^{2}+\left\|A^{-1 / 2} u\right\|_{H}^{2}\right)^{1 / 2} \\
& \leq\left\|A^{-1 / 2} B A^{-1 / 2} u-A^{-1 / 2} v\right\|_{H}+\left\|A^{-1 / 2} u\right\|_{H} \\
& \leq\left\|A^{-1 / 2} B A^{-1 / 2}\right\|\|u\|_{H}+\left\|A^{-1 / 2}\right\|\|v\|_{H}+\left\|A^{-1 / 2}\right\|\|u\|_{H} \\
& \leq\left(\left(\left\|A^{-1 / 2} B A^{-1 / 2}\right\|+\left\|A^{-1 / 2}\right\|\right)^{2}+\left\|A^{-1 / 2}\right\|^{2}\right)^{1 / 2} \cdot\left(\|u\|_{H}^{2}+\|v\|_{H}^{2}\right)^{1 / 2} \\
& \leq\left(\left\|A^{-1 / 2} B A^{-1 / 2}\right\|+2\left\|A^{-1 / 2}\right\|\right)\left(\|u\|_{H}^{2}+\|v\|_{H}^{2}\right)^{1 / 2} .
\end{aligned}
$$


Since $\mathscr{D}\left(\Lambda_{0}\right)$ is dense in $\tilde{\mathbb{H}}$, we thus have

$$
\left\|\Lambda_{0}^{-1}\right\|_{\mathscr{L}(\tilde{\mathbb{H}})} \leq\left\|A^{-1 / 2} B A^{-1 / 2}\right\|+2\left\|A^{-1 / 2}\right\|,
$$

i.e.,

$$
\left\|\Lambda^{-1}\right\|_{\mathscr{L}(\mathbb{H})} \leq\left\|A^{-1 / 2} B A^{-1 / 2}\right\|+2\left\|A^{-1 / 2}\right\| .
$$

By virtue of Corollary 3.1, we obtain the desired result.

Next we employ Lemma 3.1 to estimate the resolvent $R(i b, \Lambda), b \in \mathbb{R}$, which will play an important role in dealing with stochastic second-order Cauchy problems with delay.

Lemma 3.3. Under the same conditions as in Theorem 2.1 and Lemma 3.1, we have for every $0<c<1$,

$$
\|R(i b, \Lambda)\| \leq\left\{\begin{array}{lll}
\frac{\left\|\Lambda^{-1}\right\|}{1-c} & \text { for } & |b| \leq \frac{c}{\left\|\Lambda^{-1}\right\|}, \\
\frac{(3+\gamma) \alpha\left\|\Lambda^{-1}\right\|+c}{\alpha c} & \text { for } & |b|>\frac{c}{\left\|\Lambda^{-1}\right\|},
\end{array}\right.
$$

Proof. Since $\Lambda$ is invertible, the resolvent of $\Lambda$ is given by

$$
R(\lambda, \Lambda)=\sum_{n=1}^{\infty} \lambda^{n} \Lambda^{-(n+1)} \quad \text { for } \quad|\lambda|<\frac{1}{\left\|\Lambda^{-1}\right\|} .
$$

Moreover, for $0<c<1$, we have that

$$
\|R(\lambda, \Lambda)\| \leq\left\|\Lambda^{-1}\right\| \sum_{n=0}^{\infty}|\lambda|^{n}\left\|\Lambda^{-1}\right\|^{n}=\frac{\left\|\Lambda^{-1}\right\|}{1-c} \quad \text { for any } \quad|\lambda| \leq \frac{c}{\left\|\Lambda^{-1}\right\|} .
$$

For $|\lambda|>c /\left\|\Lambda^{-1}\right\|$, let $0<\delta<\alpha$ and we have by Lemma 3.1 that

$$
\|R(i b, \Lambda)\|<\frac{1}{\delta} \quad \text { for } \quad|b|>\frac{\alpha(3 \delta+\gamma)}{\alpha-\delta} .
$$

Now we have to look for one $\delta \in(0, \alpha)$ such that

$$
\frac{\alpha(3 \delta+\gamma)}{\alpha-\delta}=\frac{c}{\left\|\Lambda^{-1}\right\|}
$$

But this is possible when

$$
\delta=\frac{\alpha c}{\alpha(3+\gamma)\left\|\Lambda^{-1}\right\|+c}
$$

which concludes the proof.

Corollary 3.3. Under the same conditions as in Lemma 3.3, we have

$$
\|R(i b, \Lambda)\| \leq \frac{2 \alpha(3+\gamma) \kappa^{-1}+1}{\alpha}
$$

for all $b \in \mathbb{R}$ and every lower bound $\kappa>0$ of $\Lambda$.

Proof. Since $\left\|\Lambda^{-1}\right\| \leq \kappa^{-1}$, we choose $c=1 / 2$ in Lemma 3.3 and obtain

$$
\|R(i b, \Lambda)\| \leq \frac{2 \alpha(3+\gamma)\left\|\Lambda^{-1}\right\|+1}{\alpha} \leq \frac{2 \alpha(3+\gamma) \kappa^{-1}+1}{\alpha}
$$

as desired. 


\section{Asymptotic Behavior of Solutions with Delay}

Now let $r>0$ and consider a linear Cauchy problem with memory in the Hilbert space $H$,

$$
\left\{\begin{array}{l}
d\left(\frac{d u(t)}{d t}\right)+A u(t) d t=B u^{\prime}(t) d t+M u_{t} d t+N u_{t}^{\prime} d t, \quad t \geq 0 \\
u(0)=\phi_{0,1} \in V, \quad u^{\prime}(0)=\phi_{0,2} \in H \\
u_{0}=\phi_{1,1} \in L^{2}([-r, 0], V), \quad u_{0}^{\prime}=\phi_{1,2} \in L^{2}([-r, 0], H),
\end{array}\right.
$$

where $A, B$ and $V$ are given as in Section 2 and the mappings $M: W^{1,2}([-r, 0], V) \rightarrow H$, $N: W^{1,2}([-r, 0], H) \rightarrow H$ are two bounded linear operators. Here $W^{1,2}([-r, 0], V)$ and $W^{1,2}([-r, 0], H)$ are the standard Sobolev spaces of functions from $[-r, 0]$ into $V$ and $H$, respectively. We strengthen the condition on $M, N$ by assuming further that $M, N$ are given by the Riemann-Stieljes integrals of functions of bounded variation $\eta:[-r, 0] \rightarrow \mathscr{L}(V, H)$ and $\eta:[-r, 0] \rightarrow \mathscr{L}(H)$, i.e.,

$$
M(\varphi)=\int_{-r}^{0} d \eta(\theta) \varphi(\theta) \quad \forall \varphi \in W^{1,2}([-r, 0], V),
$$

and

$$
N(\varphi)=\int_{-r}^{0} d \zeta(\theta) \varphi(\theta) \quad \forall \varphi \in W^{1,2}([-r, 0], H) .
$$

For each $\lambda \in \mathbb{C}$, we define in connection with $M$ a linear operator $M\left(e^{\lambda \cdot}\right): V \rightarrow V$ by

$$
M\left(e^{\lambda \cdot}\right) x=M\left(e^{\lambda \cdot} x\right), \quad x \in V .
$$

Then it is easy to see that $M\left(e^{\lambda \cdot}\right) \in \mathscr{L}(V)$ and

$$
\left\|M\left(e^{\lambda \cdot}\right)\right\|_{\mathscr{L}(V)} \leq e^{|\lambda| r} \operatorname{Var}(\eta)_{-r}^{0}
$$

where $\operatorname{Var}(\eta)_{-r}^{0}$ is the total variation of $\eta$ on $[-r, 0]$. In a similar way, one can define and show $N\left(e^{\lambda \cdot}\right) \in \mathscr{L}(H)$ and

$$
\left\|N\left(e^{\lambda \cdot}\right)\right\|_{\mathscr{L}(H)} \leq e^{|\lambda| r} \operatorname{Var}(\zeta)_{-r}^{0} .
$$

By defining $y(t)=\left(\begin{array}{c}u(t) \\ u^{\prime}(t)\end{array}\right), y_{t}=\left(\begin{array}{c}u_{t} \\ u_{t}^{\prime}\end{array}\right)$, let us rewrite the problem (4.1) as a first-order delay differential equation in $\mathbb{H}$,

$$
\left\{\begin{array}{l}
d y(t)=\Lambda y(t) d t+F y_{t} d t, \quad t \geq 0 \\
y(0)=\phi_{0}=\left(\begin{array}{c}
\phi_{0,1} \\
\phi_{0,2}
\end{array}\right) \in \mathbb{H}, \quad y_{0}=\phi_{1}=\left(\begin{array}{c}
\phi_{1,1} \\
\phi_{1,2}
\end{array}\right) \in L^{2}([-r, 0], \mathbb{H})
\end{array}\right.
$$

where $\Lambda$ is given as in (2.2) and delay operator matrix $F=\left(\begin{array}{cc}0 & 0 \\ M & N\end{array}\right)$ is a bounded linear operator from $W^{1,2}([-r, 0], V) \times W^{1,2}([-r, 0, H)$ into $\mathbb{H}$ given by

$$
F\left(\begin{array}{l}
\varphi_{1} \\
\varphi_{2}
\end{array}\right)=\left(\begin{array}{cc}
0 & 0 \\
M & N
\end{array}\right)\left(\begin{array}{l}
\varphi_{1} \\
\varphi_{2}
\end{array}\right)=\left(\int_{-r}^{0} d \eta(\theta) \varphi_{1}(\theta)+\int_{-r}^{0} d \zeta(\theta) \varphi_{2}(\theta)\right)
$$


for any $\varphi_{1} \in W^{1,2}([-r, 0], V), \varphi_{2} \in W^{1,2}([-r, 0], H)$.

Let $\mathcal{H}_{2}$, or simply $\mathcal{H}$, denote the Hilbert space $\mathbb{H} \times L^{2}([-r, 0], \mathbb{H})$, equipped with the usual product space inner product

$$
\langle\varphi, \psi\rangle_{\mathcal{H}_{2}}:=\left\langle\varphi_{0}, \psi_{0}\right\rangle_{\mathbb{H}}+\int_{-r}^{0}\left\langle\varphi_{1}(\theta), \psi_{1}(\theta)\right\rangle_{\mathbb{H}} d \theta
$$

for any $\varphi=\left(\varphi_{0}, \varphi_{1}\right), \psi=\left(\psi_{0}, \psi_{1}\right) \in \mathcal{H}_{2}$. It may be shown that for any $\phi=\left(\phi_{0}, \phi_{1}\right) \in \mathcal{H}_{2}$, the equation (4.2) has a unique mild solution $y(t, \phi)$. For any $x \in \mathbb{H}$, we introduce the fundamental solution or Green operator $G(t):(-\infty, \infty) \rightarrow \mathscr{L}(\mathbb{H})$ of (4.2) by

$$
G(t) x= \begin{cases}y(t, \phi), & t \geq 0, \\ 0, & t<0,\end{cases}
$$

where $\phi=(x, 0), x \in \mathbb{H}$. It turns out (cf. [10]) that $G(t), t \geq 0$, is a strongly continuous one-parameter family of bounded linear operators on $\mathbb{H}$. For each function $\varphi:[-r, 0] \rightarrow \mathbb{H}$, we define its right extension function $\vec{\varphi}$ by

$$
\vec{\varphi}:[-r, \infty) \rightarrow \mathbb{H}, \quad \vec{\varphi}(t)= \begin{cases}\varphi(t), & -r \leq t \leq 0, \\ 0, & 0<t<\infty\end{cases}
$$

By virtue of (4.4), it may be shown (cf. [10]) that the mild solution $y(t, \phi)$ of (4.2) is represented explicitly by the variation of constants formula

$$
y(t)=G(t) \phi_{0}+\int_{0}^{t} G(t-s) F\left(\overrightarrow{\phi_{1}}\right)_{s} d s, \quad t \geq 0,
$$

and $y(t)=\phi_{1}(t), t \in[-r, 0)$. It is useful to introduce the so-called structure operator $S$ defined on the space $L^{2}([-r, 0] ; \mathbb{H})$ by

$$
(S \varphi)(\theta)=F \vec{\varphi}_{-\theta}, \quad \theta \in[-r, 0], \quad \forall \varphi(\cdot) \in W^{1,2}([-r, 0] ; \mathbb{H}) .
$$

It is not difficult to show that $S$ can be extended to a linear and bounded operator from $L_{r}^{2}:=L^{2}([-r, 0] ; \mathbb{H})$ into itself. Moreover, the variation of constants formula for the mild solution of (4.2) may be rewritten as

$$
\left\{\begin{array}{l}
y(t)=G(t) \phi_{0}+\int_{-r}^{0} G(t+\theta)\left(S \phi_{1}\right)(\theta) d \theta, \quad t \geq 0 \\
y(0)=\phi_{0}, y(\theta)=\phi_{1}(\theta), \quad \theta \in[-r, 0] .
\end{array}\right.
$$

The mild solution $y(t, \phi)$ of (4.2) allows us to introduce a $C_{0}$-semigroup on $\mathcal{H}_{2}$. Indeed, we define a mapping $\mathcal{S}(t), t \geq 0$, associated with $y(t, \phi)$, by

$$
\mathcal{S}(t) \phi=\left(y(t, \phi), y_{t}(\cdot, \phi)\right), \quad t \geq 0, \quad \phi \in \mathcal{H} .
$$

It can be shown (cf. [12]) that the mapping $\mathcal{S}(t), t \geq 0$, is a $C_{0}$-semigroup with some infinitesimal generator $\mathcal{A}$ on the space $\mathcal{H}_{2}$. Moreover, the operator $\mathcal{A}$ may be explicitly specified as follows. 
Proposition 4.1. The generator $\mathcal{A}$ of the $C_{0}$-semigroup $\mathcal{S}(t)$, or denote it by $e^{t \mathcal{A}}$, is described by

$$
\begin{gathered}
\mathscr{D}(\mathcal{A})=\left\{\phi=\left(\phi_{0}, \phi_{1}\right) \in \mathcal{H}_{2}: \phi_{1} \in W^{1,2}([-r, 0] ; \mathbb{H}), \phi_{1}(0)=\phi_{0} \in \mathscr{D}(\Lambda)\right\}, \\
\mathcal{A} \phi=\left(\Lambda \phi_{0}+F \phi_{1}, \frac{d \phi}{d \theta}(\theta)\right) \text { for any } \phi=\left(\phi_{0}, \phi_{1}\right) \in \mathscr{D}(\mathcal{A}) .
\end{gathered}
$$

The proof of the following proposition is referred to Proposition 2.2 in [11].

Proposition 4.2. For the Green operator $G(t), t \in \mathbb{R}$, and $C_{0}$-semigroup $e^{t \mathcal{A}}, t \geq 0$, in (4.8), the following relations are equivalent:

(i) the semigroup $e^{t \mathcal{A}}$ is exponentially stable, i.e., there exist constants $M>0$ and $\mu>0$ such that $\left\|e^{t \mathcal{A}}\right\| \leq M e^{-\mu t}, t \geq 0$.

(ii) the Green operator $G(t)$ is exponentially stable, i.e., there exist constants $M>0$ and $\mu>0$ such that $\|G(t)\| \leq M e^{-\mu t}, t \geq 0$.

Now we consider the asymptotic behavior of $C_{0}$-semigroup $e^{t \mathcal{A}}, t \geq 0$, on $\mathcal{H}$. Recall that for any $\lambda \in \mathbb{C}, F\left(e^{\lambda \cdot}\right) y:=F\left(e^{\lambda \cdot} y\right), y \in \mathbb{H}$, defines a bounded linear operator $F\left(e^{\lambda \cdot}\right)$ on $\mathbb{H}$. We first state a proposition whose proof is referred to Theorem 5.5, pp. 104-105, in [2].

Proposition 4.3. Assume that $\Lambda$ generates an exponentially stable $C_{0}$-semigroup $e^{t \Lambda}, t \geq 0$, on $\mathbb{H}$, i.e., $\omega_{g}(\Lambda)<0$, and let $a \in\left(\omega_{g}(\Lambda), 0\right]$ such that

$$
\alpha_{a, n}:=\sup _{b \in \mathbb{R}}\left\|\left[F\left(e^{(a+i b) \cdot}\right) R(a+i b, \Lambda)\right]^{n}\right\|<\infty \quad \text { for any } \quad n \in \mathbb{N} .
$$

Further, if the series $\sum_{n=0}^{\infty} \alpha_{a, n}<\infty$, then $\omega_{g}(\mathcal{A})<a \leq 0$. That is, $\mathcal{A}$ generates in this case an exponentially stable $C_{0}$-semigroup $e^{t \mathcal{A}}, t \geq 0$, on $\mathcal{H}$.

Corollary 4.1. Assume that the growth bound of $\Lambda$ satisfies $\omega_{g}(\Lambda)<0$, and let $a \in$ $\left(\omega_{g}(\Lambda), 0\right]$. If

$$
\sup _{b \in \mathbb{R}}\left\|F\left(e^{(a+i b) \cdot}\right)\right\|<\frac{1}{\sup _{b \in \mathbb{R}}\|R(a+i b, \Lambda)\|},
$$

then $\omega_{g}(\mathcal{A})<a \leq 0$.

Proof. Defining $q_{a}=\sup _{b \in \mathbb{R}}\left\|F\left(e^{(a+i b) \cdot}\right) R(a+i b, \Lambda)\right\|<1$, we obtain that $\alpha_{a, n} \leq q_{a}^{n}$, hence the series $\sum_{n=0}^{\infty} \alpha_{a, n}$ is convergent.

\section{Stochastic Systems Driven by Wiener Processes}

Let $\{\Omega, \mathscr{F}, \mathbb{P}\}$ be a probability space equipped with some filtration $\left\{\mathscr{F}_{t}\right\}_{t \geq 0}$. Let $K$ be a separable Hilbert space and $\left\{W_{Q}(t), t \geq 0\right\}$ denote a $Q$-Wiener process with respect to $\left\{\mathscr{F}_{t}\right\}_{t \geq 0}$ in $K$, defined on $\{\Omega, \mathscr{F}, \mathbb{P}\}$, with covariance operator $Q$, i.e.,

$$
\mathbb{E}\left\langle W_{Q}(t), x\right\rangle_{K}\left\langle W_{Q}(s), y\right\rangle_{K}=(t \wedge s)\langle Q x, y\rangle_{K} \text { for all } x, y \in K,
$$


where $Q$ is a positive, self-adjoint and trace class operator on $K$. We frequently call $W_{Q}(t)$, $t \geq 0$, a $K$-valued $Q$-Wiener process with respect to $\left\{\mathscr{F}_{t}\right\}_{t \geq 0}$ if the trace $\operatorname{Tr} Q<\infty$. We introduce a subspace $K_{Q}=\mathscr{R}\left(Q^{1 / 2}\right) \subset K$, the range of $Q^{1 / 2}$, which is a Hilbert space endowed with the inner product

$$
\langle u, v\rangle_{K_{Q}}=\left\langle Q^{-1 / 2} u, Q^{-1 / 2} v\right\rangle_{K} \quad \text { for any } u, v \in K_{Q}
$$

Let $X$ be a separable Hilbert space and $\mathscr{L}_{2}\left(K_{Q}, X\right)$ denote the space of all Hilbert-Schmidt operators from $K_{Q}$ into $X$. Then $\mathscr{L}_{2}\left(K_{Q}, X\right)$ turns out to be a separable Hilbert space, equipped with the norm

$$
\|\Psi\|_{\mathscr{L}_{2}\left(K_{Q}, X\right)}^{2}=\operatorname{Tr}\left[\Psi Q^{1 / 2}\left(\Psi Q^{1 / 2}\right)^{*}\right] \quad \text { for any } \Psi \in \mathscr{L}_{2}\left(K_{Q}, X\right) .
$$

For arbitrarily given $T \geq 0$, let $J(t, \omega), t \in[0, T]$, be an $\mathscr{L}_{2}\left(K_{Q}, X\right)$-valued process, and we define the following norm for arbitrary $t \in[0, T]$,

$$
|J|_{t}:=\left\{\mathbb{E} \int_{0}^{t} \operatorname{Tr}\left[J(s, \omega) Q^{1 / 2}\left(J(s, \omega) Q^{1 / 2}\right)^{*}\right] d s\right\}^{\frac{1}{2}} .
$$

In particular, we denote all $\mathscr{L}_{2}\left(K_{Q}, X\right)$-valued measurable processes $J$, adapted to the filtration $\left\{\mathscr{F}_{t}\right\}_{t \leq T}$, satisfying $|J|_{T}<\infty$ by $\mathcal{U}^{2}\left([0, T] ; \mathscr{L}_{2}\left(K_{Q}, X\right)\right)$.

Let $r>0$ and consider a semi-linear Cauchy problem with memory in the Hilbert space $H$

$$
\left\{\begin{array}{l}
d\left(\frac{d u(t)}{d t}\right)+A u(t) d t=B u^{\prime}(t) d t+M u_{t} d t+N u_{t}^{\prime} d t+R\left(u(t), u^{\prime}(t), u_{t}, u_{t}^{\prime}\right) d W(t), \quad t \geq 0 \\
u(0)=\phi_{0,1} \in V, \quad u^{\prime}(0)=\phi_{0,2} \in H \\
u_{0}=\phi_{1,1} \in L^{2}([-r, 0], V), \quad u_{0}^{\prime}=\phi_{1,2} \in L^{2}([-r, 0], H),
\end{array}\right.
$$

where $A, B, M, N$ and $V$ are given as in Section 4 and $W$ is a $Q$-Wiener process in $K$. The non-linear mapping

$$
R: V \times H \times L^{2}([-r, 0], V) \times L^{2}([-r, 0], H) \rightarrow \mathscr{L}_{2}\left(K_{Q}, H\right)
$$

is assumed to be Borel measurable and there exist constants $\alpha_{1}, \alpha_{2}>0$ and a finite measure $\kappa(\cdot)$ on $[-r, 0]$ such that

$$
\begin{aligned}
\|R(\phi)-R(\psi)\|_{\mathscr{L}_{2}\left(K_{Q}, H\right)}^{2} \leq \alpha_{1}\left(\left\|\phi_{0,1}-\psi_{0,1}\right\|_{V}^{2}+\left\|\phi_{0,2}-\psi_{0,2}\right\|_{H}^{2}\right) \\
\quad+\alpha_{2}\left(\int_{-r}^{0}\left\|\phi_{1,1}(\theta)-\psi_{1,1}(\theta)\right\|_{V}^{2} \kappa(d \theta)+\int_{-r}^{0}\left\|\phi_{1,2}(\theta)-\psi_{1,2}(\theta)\right\|_{H}^{2} \kappa(d \theta)\right)
\end{aligned}
$$

for any $\phi=\left(\phi_{0}, \phi_{1}\right)=\left(\phi_{0,1}, \phi_{0,2}, \phi_{1,1}, \phi_{1,2}\right), \psi=\left(\psi_{0}, \psi_{1}\right)=\left(\psi_{0,1}, \psi_{0,2}, \psi_{1,1}, \psi_{1,2}\right) \in V \times H \times$ $L^{2}([-r, 0], V) \times L^{2}([-r, 0], H)$. 
Our strategy here is to apply the techniques developed in the previous sections. Hence, as the first step, let us rewrite this problem into a first-order stochastic delay system. That is, by defining $y(t)=\left(\begin{array}{c}u(t) \\ u^{\prime}(t)\end{array}\right), y_{t}=\left(\begin{array}{c}u_{t} \\ u_{t}^{\prime}\end{array}\right)$ and a nonlinear mapping $L$ by

$$
L\left(\phi_{0}, \phi_{1}\right)=\left(\begin{array}{c}
0 \\
R\left(\phi_{0,1}, \phi_{0,2}, \phi_{1,1}, \phi_{1,2}\right)
\end{array}\right)
$$

for any $\phi=\left(\phi_{0}, \phi_{1}\right)=\left(\phi_{0,1}, \phi_{0,2}, \phi_{1,1}, \phi_{1,2}\right) \in V \times H \times L^{2}([-r, 0], V) \times L^{2}([-r, 0], H)$, we can transform (5.2) into a first-order stochastic system

$$
\left\{\begin{array}{l}
d y(t)=\Lambda y(t) d t+F y_{t} d t+L\left(y(t), y_{t}\right) d W(t), \quad t \geq 0 \\
y(0)=\phi_{0}=\left(\begin{array}{c}
\phi_{0,1} \\
\phi_{0,2}
\end{array}\right) \in \mathbb{H}, \quad y_{0}=\phi_{1}=\left(\begin{array}{c}
\phi_{1,1} \\
\phi_{1,2}
\end{array}\right) \in L^{2}([-r, 0], \mathbb{H}),
\end{array}\right.
$$

where $L: \mathcal{H} \rightarrow \mathscr{L}_{2}\left(K_{Q}, \mathbb{H}\right)$ is clearly Borel measurable and (15.3) implies immediately that

$$
\begin{gathered}
\|L(\phi)-L(\psi)\|_{\mathscr{L}_{2}\left(K_{Q}, \mathbb{H}\right)}^{2} \leq \alpha_{1}\left\|\phi_{0}-\psi_{0}\right\|_{\mathbb{H}}^{2}+\alpha_{2} \int_{-r}^{0}\left\|\phi_{1}(\theta)-\psi_{1}(\theta)\right\|_{L^{2}([-r, 0], \mathbb{H})}^{2} \kappa(d \theta), \\
\forall \phi=\left(\phi_{0}, \phi_{1}\right), \psi=\left(\psi_{0}, \psi_{1}\right) \in \mathcal{H} .
\end{gathered}
$$

Let $C_{b}(\mathcal{H})$ denote the set of all bounded and continuous real-valued functions on $\mathcal{H}$ and $\mathscr{P}(\mathcal{H})$ be the space of all probability measures on $(\mathcal{H}, \mathscr{B}(\mathcal{H}))$ where $\mathscr{B}(\mathcal{H})$ is the Borel $\sigma$ algebra on $\mathcal{H}$. For any mild solution $y$ of (5.4), it is well-known that $Y(t):=\left(y(t), y_{t}\right), t \geq 0$, is a Markov process in $\mathcal{H}$.

Definition 5.1. A stationary distribution for $Y(t)=\left(y(t), y_{t}\right), t \geq 0$, of equation (5.4) is defined as a probability measure $\mu \in \mathscr{P}(\mathcal{H})$ satisfying

$$
\mu(f)=\mu\left(\mathbb{P}_{t} f\right), \quad t \geq 0,
$$

where

$$
\mu(f):=\int_{\mathcal{H}} f(\phi) \mu(d \phi) \quad \text { and } \quad \mathbb{P}_{t} f(\phi):=\mathbb{E} f(Y(t, \phi)), \quad f \in C_{b}(\mathcal{H})
$$

For $\mu_{1}, \mu_{2} \in \mathscr{P}(\mathcal{H})$, define a metric on $\mathscr{P}(\mathcal{H})$ by

$$
d\left(\mu_{1}, \mu_{2}\right)=\sup _{f \in \mathcal{M}}\left|\int_{\mathcal{H}} f(\phi) \mu_{1}(d \phi)-\int_{\mathcal{H}} f(\psi) \mu_{2}(d \psi)\right|
$$

where

$$
\mathcal{M}:=\left\{f: \mathcal{H} \rightarrow \mathbb{R},|f(\phi)-f(\psi)| \leq\|\phi-\psi\|_{\mathcal{H}} \text { for any } \phi, \psi \in \mathcal{H} \text { and }|f(\cdot)| \leq 1\right\}
$$

Then it is well known (cf. [4] or [13]) that $\mathscr{P}(\mathcal{H})$ is complete under the metric $d(\cdot, \cdot)$. 
Lemma 5.1. Suppose that for any bounded subset $U$ of $\mathcal{H}$,

(i) $\lim _{t \rightarrow \infty} \sup _{\phi, \psi \in U} \mathbb{E}\|Y(t, \phi)-Y(t, \psi)\|_{\mathcal{H}}^{2}=0$;

(ii) $\sup _{t \geq 0} \sup _{\phi \in U} \mathbb{E}\|Y(t, \phi)\|_{\mathcal{H}}^{2}<\infty$.

Then, for initial data $\phi \in \mathcal{H}$, process $Y(t, \phi), t \geq 0$, has a stationary distribution.

Proof. It suffices to show that for any initial data $\phi \in \mathcal{H},\{\mathbb{P}(\phi, t, \cdot): t \geq 0\}$ is Cauchy in the space $\mathscr{P}(\mathcal{H})$ with the metric $d(\cdot, \cdot)$ in $(5.6)$. To this end, we need only show that for any fixed $\phi \in \mathcal{H}$ and $\varepsilon>0$, there exists a time $T>0$ such that

$$
d(\mathbb{P}(\phi, t+s, \cdot), \mathbb{P}(\phi, t, \cdot))=\sup _{f \in \mathcal{M}}|\mathbb{E} f(Y(t+s, \phi))-\mathbb{E} f(Y(t, \phi))| \leq \varepsilon, \quad \forall t \geq T, s>0 .
$$

Indeed, for any $f \in \mathcal{M}$ and $t, s>0$, we can obtain that

$$
\begin{array}{rl}
\mid \mathbb{E} & f(Y(t+s, \phi))-\mathbb{E} f(Y(t, \phi)) \mid \\
\quad & \left.\mid \mathbb{E}\left[\mathbb{E} f(Y(t+s, \phi)) \mid \mathscr{F}_{s}\right)\right]-\mathbb{E} f(Y(t, \phi)) \mid \\
& =\left|\int_{H} \mathbb{E} f(Y(t, \psi)) \mathbb{P}(\phi, s, d \psi)-\mathbb{E} f(Y(t, \phi))\right| \\
& \leq \int_{H}|\mathbb{E} f(Y(t, \psi))-\mathbb{E} f(Y(t, \phi))| \mathbb{P}(\phi, s, d \psi) \\
& \leq 2 \mathbb{P}\left(\phi, s, \mathcal{H}_{R}^{c}\right)+\int_{\mathcal{H}_{R}}|\mathbb{E} f(Y(t, \psi))-\mathbb{E} f(Y(t, \phi))| \mathbb{P}(\phi, s, d \psi),
\end{array}
$$

where $\mathcal{H}_{R}=\left\{\phi \in \mathcal{H}:\|\phi\|_{\mathcal{H}} \leq R\right\}$ and $\mathcal{H}_{R}^{c}=\mathcal{H}-\mathcal{H}_{R}$. By virtue of condition (i), there exists a positive number $R$ sufficiently large such that

$$
\mathbb{P}\left(\phi, s, \mathcal{H}_{R}^{c}\right)<\frac{\varepsilon}{4}, \quad \forall s>0 .
$$

On the other hand, by virtue of condition (ii), there exists a time $T_{2}>0$ such that

$$
\sup _{f \in \mathcal{M}}|\mathbb{E} f(Y(t, \psi))-\mathbb{E} f(Y(t, \phi))| \leq \frac{\varepsilon}{2}, \quad t \geq T_{2} .
$$

Hence, substituting (5.21), (5.22) into (5.20) immediately yields that

$$
|\mathbb{E} f(Y(t+s, \phi))-\mathbb{E} f(Y(t, \phi))| \leq \varepsilon, \quad t \geq T_{2}, s>0 .
$$

Since function $f \in \mathcal{M}$ is arbitrary, we can get the desired result (5.7). Hence, the transition probability $\mathbb{P}(\phi, t, \cdot)$ of $Y(t, \phi)$ converges weakly to some $\mu \in \mathscr{P}(\mathcal{H})$. On the other hand, for any $f \in C_{b}(\mathcal{H})$, one has by the Markovian property of $Y(t, \phi), t \geq 0$ that

$$
\mathbb{P}_{t+s} f(\phi)=\mathbb{P}_{t} \mathbb{P}_{s} f(\phi), \quad t, s \geq 0, \phi \in \mathcal{H}
$$

Hence, for any fixed $t \geq 0$, as $s \rightarrow \infty$, it follows that

$$
\mu(f)=\mu\left(\mathbb{P}_{t} f\right), \quad f \in C_{b}(\mathcal{H}) .
$$

That is, $\mu$ is a stationary distribution for $Y(t)=\left(y(t), y_{t}\right), t \geq 0$, of equation (5.4) provided that (i) and (ii) above hold. The proof is thus complete.

Recall that $e^{t \mathcal{A}}, t \geq 0$, is the $C_{0}$-semigroup on $\mathcal{H}$ given in Proposition 4.1. 
Theorem 5.1. Assume that $\left\|e^{t \mathcal{A}}\right\| \leq M e^{-\gamma t}, M \geq 1, \gamma>0$ for all $t \geq 0$. Suppose that (5.5) holds and

$$
2 \gamma>3 M^{2}\left(\alpha_{1}+\alpha_{2} e^{2 \gamma r} \kappa([-r, 0])\right),
$$

then there is a unique stationary distribution $\mu \in \mathscr{P}(\mathcal{H})$ for $Y(t)=\left(y(t), y_{t}\right), t \geq 0$, of (5.4).

Proof. By Lemma 5.1, we need only verify the following assertions: for any bounded subset $U$ of $\mathcal{H}$

(i) $\lim _{t \rightarrow \infty} \sup _{\phi, \psi \in U} \mathbb{E}\|Y(t, \phi)-Y(t, \psi)\|_{\mathcal{H}}^{2}=0$;

(ii) $\sup _{t \geq 0} \sup _{\phi \in U} \mathbb{E}\|Y(t, \phi)\|_{\mathcal{H}}^{2}<\infty$.

First, it is known that the mild solution of (5.4) can be represented explicitly as

$$
y(t, \phi)=G(t) \phi_{0}+\int_{-r}^{0} G(t+\theta) S \phi_{1}(\theta) d \theta+\int_{0}^{t} G(t-s) L\left(y(s, \phi), y_{s}(\phi)\right) d W(s),
$$

where $G(\cdot)$ is the fundamental solution of (5.4) and $S \in \mathscr{L}\left(L_{r}^{2}\right)$ is the associated structure operator. To proceed further, let us consider the difference of two mild solutions of (5.4) with distinct initial data $\phi, \psi \in \mathcal{H}$ :

$$
\Sigma(t, \phi, \psi)=y(t, \phi)-y(t, \psi), \quad t \geq 0 .
$$

Note that, by assumption, we have according to Proposition 4.2 that $\|G(t)\| \leq M e^{-\gamma t}$, $M \geq 1, \gamma>0$ for all $t \geq 0$. By virtue of (5.5), (5.12) and Itô isometry, it follows that

$$
\begin{aligned}
\mathbb{E} & \|\Sigma(t, \phi, \psi)\|_{\mathbb{H}}^{2} \\
\leq & 3 \mathbb{E}\left\{\left\|G(t)\left(\phi_{0}-\psi_{0}\right)\right\|_{\mathbb{H}}^{2}+\left\|\int_{-r}^{0} G(t+\theta) S\left(\phi_{1}(\theta)-\psi_{1}(\theta)\right) d \theta\right\|_{\mathbb{H}}^{2}\right. \\
& \left.+\left\|\int_{0}^{t} G(t-s)\left[L\left(y(s, \phi), y_{s}(\phi)\right)-L\left(y(s, \psi), y_{s}(\psi)\right)\right] d W(s)\right\|_{\mathbb{H}}^{2}\right\} \\
\leq & 3\left(M^{2}+M^{2}\|S\|_{\mathscr{L}\left(L_{r}^{2}\right)}^{2} r e^{2 \gamma r}\right) e^{-2 \gamma t}\|\phi-\psi\|_{\mathcal{H}}^{2}+3 M^{2} \alpha_{1} \int_{0}^{t} e^{-2 \gamma(t-s)} \mathbb{E}\|y(s, \phi)-y(s, \psi)\|_{\mathbb{H}}^{2} d s \\
& +3 M^{2} \alpha_{2} \int_{0}^{t} e^{-2 \gamma(t-s)} \int_{-r}^{0} \mathbb{E}\|y(s+\theta, \phi)-y(s+\theta, \psi)\|_{\mathbb{H}}^{2} \kappa(d \theta) d s, \quad t \geq 0 .
\end{aligned}
$$

On the other hand, it is easy to see that for any $t \geq 0$,

$$
\begin{aligned}
& \int_{0}^{t} e^{-2 \gamma(t-s)} \int_{-r}^{0} \mathbb{E}\|y(s+\theta, \phi)-y(s+\theta, \psi)\|_{\mathbb{H}}^{2} \kappa(d \theta) d s \\
& \leq \int_{-r}^{0} \int_{-r}^{t} e^{-2 \gamma(t-s+\theta)} \mathbb{E}\|y(s, \phi)-y(s, \psi)\|_{\mathbb{H}}^{2} d s \kappa(d \theta) \\
& \leq e^{2 \gamma r} \kappa([-r, 0])\left(\|\phi-\psi\|_{\mathcal{H}}^{2}+\int_{0}^{t} e^{-2 \gamma(t-s)} \mathbb{E}\|y(s, \phi)-y(s, \psi)\|_{\mathbb{H}}^{2} d s\right) .
\end{aligned}
$$


Substituting (5.14) into (5.13), one can have that for $t \geq 0$,

$$
\mathbb{E}\|y(t, \phi)-y(t, \psi)\|_{\mathbb{H}}^{2} \leq C_{1} e^{-2 \gamma t}\|\phi-\psi\|_{\mathcal{H}}^{2}+C_{2} \int_{0}^{t} e^{-2 \gamma(t-s)}\|y(s, \phi)-y(s, \psi)\|_{\mathbb{H}}^{2} d s,
$$

where

$$
C_{1}=3 M^{2}\left(1+\|S\|_{\mathscr{L}\left(L_{r}^{2}\right)}^{2} r e^{2 \gamma r}\right)+3 M^{2} \alpha_{2} e^{2 \gamma r} \kappa([-r, 0])>0
$$

and

$$
C_{2}=3 M^{2}\left(\alpha_{1}+\alpha_{2} e^{2 \gamma r} \kappa([-r, 0])\right)>0 .
$$

Now, by multiplying $e^{2 \gamma t}$ on both sides of (5.15), we obtain for any $t \geq 0$ that

$$
e^{2 \gamma t} \mathbb{E}\|y(t, \phi)-y(t, \psi)\|_{\mathbb{H}}^{2} \leq C_{1}\|\phi-\psi\|_{\mathcal{H}}^{2}+C_{2} \int_{0}^{t} e^{2 \gamma s} \mathbb{E}\|y(s, \phi)-y(s, \psi)\|_{\mathbb{H}}^{2} d s .
$$

Hence, letting $\alpha=2 \gamma-3 M^{2}\left(\alpha_{1}+\alpha_{2} e^{2 \gamma r} \kappa([-r, 0])\right)>0$ and using the well-known Gronwall lemma, we have

$$
\mathbb{E}\|y(s, \phi)-y(s, \psi)\|_{\mathbb{H}}^{2} \leq C_{1}\|\phi-\psi\|_{\mathcal{H}}^{2} e^{-\alpha t}, \quad t \geq 0 .
$$

Further, we may obtain that for all $t \geq 0$,

$$
\begin{aligned}
\mathbb{E} \int_{-r}^{0}\|y(t+\theta, \phi)-y(t+\theta, \psi)\|_{\mathbb{H}}^{2} d \theta & \leq C_{1} \int_{-r}^{0}\|\phi-\psi\|_{\mathcal{H}}^{2} e^{-\alpha(t+\theta)} d \theta \\
& \leq r C_{1}\|\phi-\psi\|_{\mathcal{H}}^{2} e^{-\alpha(t-r)} .
\end{aligned}
$$

Hence, the relation (i) holds.

Next, we show that (ii) is also valid. To this end, first note that

$$
(a+b)^{2} \leq(1+\varepsilon) a^{2}+\left(1+\frac{1}{\varepsilon}\right) b^{2} \quad \text { for any } a, b \geq 0 \text { and } \varepsilon>0 .
$$

Then by using (5.12), (5.17) we can utilize Itô's isometry and carry out a similar argument to (5.14) to have that for $t \geq 0$,

$$
\begin{aligned}
\mathbb{E} & \|y(t, \phi)\|_{\mathbb{H}}^{2} \\
\leq & 3\left\{\left\|G(t) \phi_{0}\right\|_{\mathbb{H}}^{2}+\left\|\int_{-r}^{0} G(t-s) S \phi_{1}(\theta) d \theta\right\|_{\mathbb{H}}^{2}+\mathbb{E} \int_{0}^{t}\left\|G(t-s) L\left(y(s), y_{s}\right)\right\|_{\mathscr{L}_{2}\left(K_{Q}, \mathbb{H}\right)}^{2} d s\right\} \\
\leq & 3 M^{2}\left(1+\|S\|_{\mathscr{L}\left(L_{r}^{2}\right)}^{2} r e^{2 \gamma r}\right) e^{-2 \gamma t}\|\phi\|_{\mathcal{H}}^{2}+3 M^{2} \int_{0}^{t} e^{-2 \gamma(t-s)}\left((1+\varepsilon) \alpha_{1} \mathbb{E}\|y(s, \phi)\|_{\mathbb{H}}^{2}\right. \\
& \left.+(1+\varepsilon) \alpha_{2} \int_{-r}^{0} \mathbb{E}\|y(s+\theta, \phi)\|_{\mathbb{H}}^{2} \kappa(d \theta)+\left(1+\frac{1}{\varepsilon}\right)\|L(0,0)\|_{\mathscr{L}_{2}\left(K_{Q}, \mathbb{H}\right)}^{2}\right) d s \\
\leq & 3 M^{2}\left(1+\|S\|_{\mathscr{L}\left(L_{r}^{2}\right)}^{2} r e^{2 \gamma r}+e^{2 \gamma r} \kappa([-r, 0])(1+\varepsilon) \alpha_{2} e^{-2 \gamma t}\|\phi\|_{\mathcal{H}}^{2}\right. \\
& +\frac{3 M^{2}}{2 \gamma}\left(1+\frac{1}{\varepsilon}\right)\|L(0,0)\|_{\mathscr{L}_{2}\left(K_{Q}, \mathbb{H}\right)}^{2}+3 M^{2} \alpha_{1}(1+\varepsilon) \int_{0}^{t} e^{-2 \gamma(t-s)} \mathbb{E}\|y(s, \phi)\|_{\mathbb{H}}^{2} d s \\
& +3 M^{2} \alpha_{2} e^{2 \gamma r}(1+\varepsilon) \kappa([-r, 0]) \int_{0}^{t} e^{-2 \gamma(t-s)} \mathbb{E}\|y(s, \phi)\|_{\mathbb{H}}^{2} d s .
\end{aligned}
$$


By choosing $\varepsilon>0$ sufficiently small and using condition (5.11) and the well-known Gronwall lemma, we thus obtain

$$
\sup _{t \geq 0} \mathbb{E}\|y(t, \phi)\|_{\mathbb{H}}^{2} \leq C_{3}\|\phi\|_{\mathcal{H}}^{2}+\frac{3 M^{2}}{2 \gamma}\left(1+\frac{1}{\varepsilon}\right)\|L(0,0)\|_{\mathscr{L}_{2}\left(K_{Q}, \mathbb{H}\right)}^{2}<\infty
$$

where

$$
C_{3}=3 M^{2}\left(1+\|S\|_{\mathscr{L}\left(L_{r}^{2}\right)}^{2} r e^{2 \gamma r}+\left(\alpha_{1}+\alpha_{2}\right)(1+\varepsilon) e^{2 \gamma r} \kappa([-r, 0])\right)>0 .
$$

By carrying out a similar argument to that of (5.17) and taking (5.18) into account, we easily have

$$
\sup _{t \geq 0} \mathbb{E} \int_{-r}^{0}\|y(t+\theta, \phi)\|_{\mathbb{H}}^{2} d \theta \leq r C_{3} e^{\alpha r}\|\phi\|_{\mathcal{H}}^{2}+\frac{3 M^{2}}{2 \gamma}\left(1+\frac{1}{\varepsilon}\right)\|L(0,0)\|_{\mathscr{L}_{2}\left(K_{Q}, \mathbb{H}\right)}^{2},
$$

and the claim (ii) is thus verified. Last, (5.19) and (5.20) together imply that

$$
\sup _{t \geq 0} \mathbb{E}\|Y(t, \phi)\|_{\mathcal{H}}^{2} \leq C_{3}\left(1+r e^{\alpha r}\right)\|\phi\|_{\mathcal{H}}^{2}+\frac{3 M^{2}}{2 \gamma}\left(1+\frac{1}{\varepsilon}\right)\|L(0,0)\|_{\mathscr{L}_{2}\left(K_{Q}, \mathbb{H}\right)}^{2}<\infty .
$$

Employing the invariance of $\pi \in \mathscr{P}(\mathcal{H})$ and integrating with respect to $\pi$ on both sides of (5.21) lead to

$$
\pi\left(\|\cdot\|_{\mathcal{H}}\right)<\infty
$$

Now we show the uniqueness of stationary distributions. If $\pi^{\prime} \in \mathscr{P}(\mathcal{H})$ is another stationary distribution for $Y(t)=\left(y(t), y_{t}\right), t \geq 0$, of equation (5.4). Let $f \in C_{L B}(\mathcal{H})$, the family of all bounded and Lipschitz continuous functions on $\mathcal{H}$. Then by virtue of (5.16), (5.17), (5.22), Hölder's inequality and the invariance of $\pi(\cdot), \pi^{\prime}(\cdot) \in \mathscr{P}(\mathcal{H})$, it follows that

$$
\left|\pi(f)-\pi^{\prime}(f)\right| \leq \int_{\mathcal{H} \times \mathcal{H}}\left|\mathbb{P}_{t} f(\phi)-\mathbb{P}_{t} f(\psi)\right| \pi(d \phi) \pi^{\prime}(d \psi) \leq C_{4} e^{-\alpha t}, \quad t \geq 0,
$$

for some constant $C_{4}>0$. This implies the uniqueness of stationary distributions by letting $t \rightarrow \infty$ in (5.23). The proof is thus complete.

\section{Systems Driven by Lévy Jump Processes}

First, let $Z$ be a $K$-valued Lévy process with its Lévy triple $(0, Q, \nu)$. For $t>0$ and $\Gamma \in \mathscr{B}(K-\{0\})$, we define a Poisson random measure generated by $Z(t)$ as

$$
N(t, \Gamma)=\sum_{s \in(0, t]} \mathbf{1}_{\Gamma}(\Delta Z(s))
$$

where $\Delta Z(t):=Z(t)-Z(t-)$ for $t \geq 0$ and the compensated Poisson random measure is given by

$$
\tilde{N}(t, \Gamma)=N(t, \Gamma)-t \nu(\Gamma), \quad t \geq 0, \quad \Gamma \in \mathscr{B}(K-\{0\})
$$


It is well known that the Lévy process $Z(t)$ has the following Lévy-Itô decomposition

$$
Z(t)=W_{Q}(t)+\int_{K-\{0\}} z \tilde{N}(t, d z)
$$

with $\operatorname{Tr}(Q)<\infty$.

Consider a second-order stochastic retarded differential equation driven by the Lévy process $Z$ in $H$, i.e., for $t \geq 0$,

$$
\left\{\begin{array}{l}
d\left(\frac{d u(t)}{d t}\right)+A u(t) d t=B u^{\prime}(t) d t+M u_{t} d t+N u_{t}^{\prime} d t+R\left(u(t-), u^{\prime}(t-), u_{t-}, u_{t-}^{\prime}\right) d Z(t), \\
u(0)=\phi_{0,1} \in V, \quad u^{\prime}(0)=\phi_{0,2} \in H \\
u_{0}=\phi_{1,1} \in L^{2}([-r, 0], V), \quad u_{0}^{\prime}=\phi_{1,2} \in L^{2}([-r, 0], H),
\end{array}\right.
$$

where $u(t-)=\lim _{s \uparrow t} u(s), u_{t-}(\theta):=\lim _{s \uparrow t} u(s+\theta)$, for $t \geq 0$ and $\theta \in[-r, 0]$, and the non-linear mapping

$$
R: V \times H \times L^{2}([-r, 0], V) \times L^{2}([-r, 0], H) \rightarrow \mathscr{L}(K, H)
$$

is assumed to be Borel measurable and there exist constants $\alpha_{1}, \alpha_{2}>0$ and a finite measure $\kappa(\cdot)$ on $[-r, 0]$ such that

$$
\begin{aligned}
& \|R(\phi)-R(\psi)\|_{\mathscr{L}(K, H)}^{2} \leq \alpha_{1}\left(\left\|\phi_{0,1}-\psi_{0,1}\right\|_{V}^{2}+\left\|\phi_{0,2}-\psi_{0,2}\right\|_{H}^{2}\right) \\
& \quad+\alpha_{2}\left(\int_{-r}^{0}\left\|\phi_{1,1}(\theta)-\psi_{1,1}(\theta)\right\|_{V}^{2} \kappa(d \theta)+\int_{-r}^{0}\left\|\phi_{1,2}(\theta)-\psi_{1,2}(\theta)\right\|_{H}^{2} \kappa(d \theta)\right)
\end{aligned}
$$

for any $\phi=\left(\phi_{0}, \phi_{1}\right)=\left(\phi_{0,1}, \phi_{0,2}, \phi_{1,1}, \phi_{1,2}\right), \psi=\left(\psi_{0}, \psi_{1}\right)=\left(\psi_{0,1}, \psi_{0,2}, \psi_{1,1}, \psi_{1,2}\right) \in V \times H \times$ $L^{2}([-r, 0], V) \times L^{2}([-r, 0], H)$. Defining $y(t)=\left(\begin{array}{c}u(t) \\ u^{\prime}(t)\end{array}\right), y_{t}=\left(\begin{array}{c}u_{t} \\ u_{t}^{\prime}\end{array}\right)$ and a nonlinear mapping $L$,

$$
L\left(\phi_{0}, \phi_{1}\right)=\left(\begin{array}{c}
0 \\
R\left(\phi_{0,1}, \phi_{0,2}, \phi_{1,1}, \phi_{1,2}\right)
\end{array}\right)
$$

for any $\phi=\left(\phi_{0}, \phi_{1}\right)=\left(\phi_{0,1}, \phi_{0,2}, \phi_{1,1}, \phi_{1,2}\right) \in V \times H \times L^{2}([-r, 0], V) \times L^{2}([-r, 0], H)$, we can transform (6.2) into a first-order stochastic system in $\mathbb{H}$

$$
\left\{\begin{array}{l}
d y(t)=\Lambda y(t) d t+F y_{t} d t+L\left(y(t-), y_{t-}\right) d Z(t), \quad t \geq 0, \\
y(0)=\phi_{0}=\left(\begin{array}{l}
\phi_{0,1} \\
\phi_{0,2}
\end{array}\right) \in \mathbb{H}, \quad y_{0}=\phi_{1}=\left(\begin{array}{c}
\phi_{1,1} \\
\phi_{1,2}
\end{array}\right) \in L^{2}([-r, 0], \mathbb{H}),
\end{array}\right.
$$

where $L: \mathcal{H} \rightarrow \mathscr{L}(K, \mathbb{H})$ is clearly Borel measurable and (6.3) implies immediately that

$$
\begin{gathered}
\|L(\phi)-L(\psi)\|_{\mathscr{L}(K, \mathbb{H})}^{2} \leq \alpha_{1}\left\|\phi_{0}-\psi_{0}\right\|_{\mathbb{H}}^{2}+\alpha_{2} \int_{-r}^{0}\left\|\phi_{1}(\theta)-\psi_{1}(\theta)\right\|_{L^{2}([-r, 0], \mathbb{H})}^{2} \kappa(d \theta), \\
\forall \phi=\left(\phi_{0}, \phi_{1}\right), \psi=\left(\psi_{0}, \psi_{1}\right) \in \mathcal{H} .
\end{gathered}
$$


Theorem 6.1. Assume that $\left\|e^{t \mathcal{A}}\right\| \leq M e^{-\gamma t}, M \geq 1, \gamma>0$ for all $t \geq 0$. Suppose that (6.3) holds and

$$
\int_{z \neq 0}\|z\|_{K}^{2} \nu(d z)<\infty
$$

and further

$$
2 \gamma>3 M^{2}\left(\operatorname{Tr}(Q)+\int_{z \neq 0}\|z\|_{K}^{2} \nu(d z)\right)\left(\alpha_{1}+\alpha_{2} e^{2 \gamma r} \kappa([-r, 0])\right),
$$

then there exists a unique stationary distribution $\mu \in \mathscr{P}(\mathcal{H})$ for $Y(t)=\left(y(t), y_{t}\right), t \geq 0$, of (6.2).

Proof. By the variation-of-constants formula, one has

$$
y(t, \phi)=G(t) \phi_{0}+\int_{-r}^{0} G(t+\theta) S \phi_{1}(\theta) d \theta+\int_{0}^{t} G(t-s) L\left(y(s-), y_{s-}\right) d Z(s) .
$$

Thus, by virtue of (6.1), we have

$$
\begin{aligned}
y(t, \phi)= & G(t) \phi_{0}+\int_{-r}^{0} G(t+\theta) S \phi_{1}(\theta) d \theta+\int_{0}^{t} G(t-s) L\left(y(s), y_{s}\right) d W_{Q}(s) \\
& +\int_{0}^{t} \int_{K-\{0\}} G(t-s) L\left(y(s-), y_{s-}\right) z \tilde{N}(d s, d z)
\end{aligned}
$$

Carrying out a similar argument to that of (5.18) and taking (6.6) into account, one can easily obtain that

$$
\begin{aligned}
\mathbb{E}\|y(t, \phi)\|_{\mathbb{H}}^{2} \leq & 6\left\{\left\|G(t) \phi_{0}\right\|_{\mathbb{H}}^{2}+\left\|\int_{-r}^{0} G(t-s) S \phi_{1}(\theta)\right\|_{\mathbb{H}}^{2} d \theta\right\} \\
& +3 \mathbb{E}\left\|\int_{0}^{t} G(t-s) L\left(y(s), y_{s}\right) d W_{Q}(s)\right\|_{\mathbb{H}}^{2} \\
& +3 \mathbb{E}\left\|\int_{0}^{t} \int_{z \neq 0} G(t-s) L\left(y(s-), y_{s-}\right) z \tilde{N}(d s, d z)\right\|_{\mathbb{H}}^{2} \\
:= & I_{1}(t)+I_{2}(t)+I_{3}(t) .
\end{aligned}
$$

Hence, we have by using (6.5) that for any $t \geq 0$,

$$
\begin{gathered}
I_{1}(t) \leq 6 M^{2}\left(1+\|S\|_{\mathscr{L}\left(L_{r}^{2}\right)}^{2} r e^{2 \gamma r}\right) e^{-2 \gamma t}\|\phi\|_{\mathcal{H}}^{2} \\
I_{2}(t) \leq 3 M^{2} \operatorname{Tr}(Q) \int_{0}^{t} e^{-2 \gamma(t-s)}\left\|L\left(y(s), y_{s}\right)\right\|_{\mathscr{L}(K, \mathbb{H})}^{2} d s \\
\leq 6 M^{2} \operatorname{Tr}(Q) \alpha_{1} \int_{0}^{t} e^{-2 \gamma(t-s)} \mathbb{E}\|y(s)\|_{\mathbb{H}}^{2} d s \\
+6 M^{2} \operatorname{Tr}(Q) \alpha_{2} \int_{0}^{t} e^{-2 \gamma(t-s)} \int_{-r}^{0} \mathbb{E}\|y(s+\theta)\|_{\mathbb{H}}^{2} \kappa(d \theta) d s \\
+6 M^{2} \operatorname{Tr}(Q) \int_{0}^{t} e^{-2 \gamma(t-s)}\|L(0,0)\|_{\mathscr{L}(K, \mathbb{H})}^{2} d s
\end{gathered}
$$


and

$$
\begin{aligned}
I_{3}(t) \leq & 3 M^{2} \int_{0}^{t} \int_{z \neq 0} e^{-2 \gamma(t-s)}\left\|L\left(y(s-), y_{s-}\right)\right\|_{\mathscr{L}(K, \mathbb{H})}^{2}\|z\|_{H}^{2} N(d s, d z) \\
\leq & 6 M^{2} \int_{z \neq 0}\|z\|_{H}^{2} \nu(d z)\left(\alpha_{1} \int_{0}^{t} e^{-2 \gamma(t-s)} \mathbb{E}\|y(s)\|_{\mathbb{H}}^{2} d s\right. \\
& \left.+\alpha_{2} \int_{0}^{t} e^{-2 \gamma(t-s)} \int_{-r}^{0} \mathbb{E}\|y(s+\theta)\|_{\mathbb{H}}^{2} \kappa(d \theta) d s+\int_{0}^{t} e^{-2 \gamma(t-s)}\|L(0,0)\|_{\mathscr{L}(K, \mathbb{H})}^{2} d s\right) .
\end{aligned}
$$

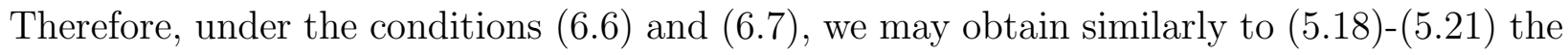
following relation

$$
\sup _{t \geq 0} \mathbb{E}\|y(t, \phi)\|_{\mathbb{H}}^{2}<\infty
$$

which shows the conclusion (ii) in Lemma 5.1. Finally, the desired assertion (ii) in Lemma 5.1 can be concluded by imitating the arguments of Theorem 5.1.

It is well-known that for a Lévy jump process, there is, in general, no finite second moment (6.6). As a result, there is generally no Itô's isometry as shown in Theorem 6.1. To go around this difficulty, let us formulate our system in a product Banach space $\mathcal{H}_{1}=\mathbb{H} \times L^{1}([-r, 0], \mathbb{H})$, equipped with the norm

$$
\|\phi\|_{\mathcal{H}_{1}}=\left\|\phi_{0}\right\|_{\mathbb{H}}+\int_{-r}^{0}\left\|\phi_{1}(\theta)\right\|_{\mathbb{H}} d \theta, \quad \phi=\left(\phi_{0}, \phi_{1}\right) \in \mathcal{H}_{1} .
$$

Consider a linear Cauchy problem with memory in the Hilbert space $H$,

$$
\left\{\begin{array}{l}
d\left(\frac{d u(t)}{d t}\right)+A u(t) d t=B u^{\prime}(t) d t+M u_{t} d t+N u_{t}^{\prime} d t+R d Z(t), \quad t \geq 0 \\
u(0)=\phi_{0,1} \in V, \quad u^{\prime}(0)=\phi_{0,2} \in H \\
u_{0}=\phi_{1,1} \in L^{2}([-r, 0], V), \quad u_{0}^{\prime}=\phi_{1,2} \in L^{2}([-r, 0], H),
\end{array}\right.
$$

where $A, B, M, N$ and $V$ are given as in Section $4, Z$ is a $K$-valued Lévy process with its Lévy triple $(0,0, \nu)$ and $R \in \mathscr{L}(K, H)$. Let us rewrite this problem into a first-order stochastic delay equation. Precisely, by defining $y(t)=\left(\begin{array}{c}u(t) \\ u^{\prime}(t)\end{array}\right)$, so $y_{t}=\left(\begin{array}{l}u_{t} \\ u_{t}^{\prime}\end{array}\right)$ and a bounded linear operator $L \in \mathscr{L}(K, \mathbb{H})$ by

$$
L=\left(\begin{array}{c}
0 \\
R
\end{array}\right): K \rightarrow \mathbb{H}, \quad L x=\left(\begin{array}{c}
0 \\
R x
\end{array}\right), x \in K,
$$

we can transform (6.11) into a first-order stochastic system

$$
\left\{\begin{array}{l}
d y(t)=\Lambda y(t) d t+F y_{t} d t+L d Z(t), \quad t \geq 0 \\
y(0)=\phi_{0}, y_{0}=\phi_{1}, \phi=\left(\phi_{0}, \phi_{1}\right) \in \mathcal{H}_{1}
\end{array}\right.
$$

Note that $\|L\|=\|R\|$. Indeed, for any $x \in K$,

$$
\|L x\|_{\mathbb{H}}^{2}=\|R x\|_{H}^{2} \leq\|R\|^{2}\|x\|_{K}^{2},
$$


i.e., $\|L\| \leq\|R\|$. On the other hand, by definition, it is true that

$$
\|R x\|_{H}^{2}=\|L x\|_{\mathbb{H}}^{2} \leq\|L\|^{2}\|x\|_{K}^{2}, \quad \forall x \in K,
$$

that is, $\|R\| \leq\|L\|$. Hence, $\|R\|=\|L\|$.

Theorem 6.2. Suppose that the Green operator $G(t)$ is exponentially stable, i.e., $\|G(t)\| \leq$ $M e^{-\gamma t}, M \geq 1, \gamma>0$ for all $t \geq 0$. Assume further that

$$
\int_{\|z\|_{K}>1}\|z\|_{K} \nu(d z)<\infty
$$

then there exists a unique stationary distribution $\mu \in \mathscr{P}(\mathcal{H})$ for $Y(t)=\left(y(t), y_{t}\right), t \geq 0$, of (6.12).

Proof. We need only verify the following assertions: for any bounded subset $U$ of $\mathcal{H}_{1}$,

(i) $\lim _{t \rightarrow \infty} \sup _{\phi, \psi \in U} \mathbb{E}\|Y(t, \phi)-Y(t, \psi)\|_{\mathcal{H}_{1}}=0$;

(ii) $\sup _{t \geq 0} \sup _{\phi \in U} \mathbb{E}\|Y(t, \phi)\|_{\mathcal{H}_{1}}<\infty$.

By the well-known Lévy-Itô decomposition theorem, one can get

$$
Z(t)=\int_{\|z\|_{K} \leq 1} z \tilde{N}(t, z)+\int_{\|z\|_{K}>1} z N(t, d z), \quad t \geq 0 .
$$

Hence, according to the variation-of-constants formula the mild solution of (6.12) is given by

$$
\begin{aligned}
y(t, \phi)= & G(t) \phi_{0}+\int_{-r}^{0} G(t+\theta) S \phi_{1}(\theta) d \theta+\int_{0}^{t} \int_{\|z\|_{K} \leq 1} G(t-s) L z \tilde{N}(d s, d z) \\
& +\int_{0}^{t} \int_{\|z\|_{K}>1} G(t-s) L z N(d s, d z) \\
=: & \sum_{j=1}^{4} I_{j}(t),
\end{aligned}
$$

By assumption, it is immediate to see that

$$
\sup _{t \geq 0} \mathbb{E}\left(\left\|I_{1}(t)\right\|_{\mathbb{H}}+\left\|I_{2}(t)\right\|_{\mathbb{H}}\right) \leq M\left(1+r\|S\|_{\mathscr{L}\left(L_{r}^{2}\right)}\right)\|\phi\|_{\mathcal{H}_{1}}<\infty .
$$

Note from the Hölder inequality, Itô's isometry and the uniform boundedness of $G(t), t \geq 0$, that

$$
\begin{aligned}
\sup _{t \geq 0} \mathbb{E}\left\|I_{3}(t)\right\|_{\mathbb{H}} & \leq\left(\sup _{t \geq 0} \int_{0}^{t}\|G(t-s) L\|^{2} d s \int_{\|z\|_{K} \leq 1}\|z\|_{K}^{2} \nu(d z)\right)^{1 / 2} \\
& \leq\left(\frac{M^{2}\|R\|^{2}}{2 \gamma} \int_{\|z\|_{K} \leq 1}\|z\|_{K}^{2} \nu(d z)\right)^{1 / 2}<\infty
\end{aligned}
$$


since $\nu(\cdot)$ is a Lévy measure. On the other hand, by assumption it follows that

$$
\begin{aligned}
\sup _{t \geq 0} \mathbb{E}\left\|I_{4}(t)\right\|_{\mathbb{H}} & \leq \int_{\|z\|_{K}>1}\|z\|_{K} \nu(d z) \sup _{t \geq 0}\left(\int_{0}^{t}\|G(t-s) L\| d s\right) \\
& =\frac{M\|R\|}{\gamma} \int_{\|z\|_{K}>1}\|z\|_{K} \nu(d z)<\infty .
\end{aligned}
$$

Hence, (6.13)-(6.16) yield the relation

$$
\sup _{t \geq 0} \mathbb{E}\|y(t, \phi)\|_{\mathbb{H}}=M\left(1+r\|S\|_{\mathscr{L}\left(L_{r}^{2}\right)}\right)\|\phi\|_{\mathcal{H}_{1}}+C<\infty \text { for some } C>0 .
$$

From (6.17), it is easy to have that

$$
\sup _{t \geq 0} \int_{-r}^{0} \mathbb{E}\|y(t+\theta, \phi)\|_{\mathbb{H}} d \theta \leq r\left(M\left(1+r\|S\|_{\mathscr{L}\left(L_{r}^{2}\right)}\right)\|\phi\|_{\mathcal{H}_{1}}+C\right)<\infty,
$$

which, in addition to (6.17), immediately implies that

$$
\sup _{t \geq 0} \sup _{\phi \in U} \mathbb{E}\|Y(t, \phi)\|_{\mathcal{H}_{1}}<\infty .
$$

On the other hand, for $\phi, \psi \in U$, it follows from (6.13) that

$$
y(t, \phi)-y(t, \psi)=G(t)\left(\phi_{0}-\psi_{0}\right)+\int_{-r}^{0} G(t+\theta) S\left(\phi_{1}(\theta)-\psi_{1}(\theta)\right) d \theta,
$$

which implies that

$$
\begin{aligned}
\sup _{\phi, \psi \in U} \mathbb{E} & \|y(t, \phi)-y(t, \psi)\|_{\mathbb{H}} \\
& \leq \sup _{\phi, \psi \in U}\left(M\left\|\phi_{0}-\psi_{0}\right\|_{\mathbb{H}} e^{-\gamma t}+M e^{-\gamma t} r\|S\|_{\mathscr{L}\left(L_{r}^{2}\right)} \int_{-r}^{0}\left\|\phi_{1}(\theta)-\psi_{1}(\theta)\right\|_{\mathbb{H}} d \theta\right) \\
& \leq \sup _{\phi, \psi \in U} M\left(1+r\|S\|_{\mathscr{L}\left(L_{r}^{2}\right)}\right)\|\phi-\psi\|_{\mathcal{H}_{1}} e^{-\gamma t} \\
& \rightarrow 0 \quad \text { as } t \rightarrow \infty .
\end{aligned}
$$

This further implies that

$$
\begin{array}{rl}
\sup _{\phi, \psi \in U} & \mathbb{E}\|Y(t, \phi)-Y(t, \psi)\|_{\mathcal{H}_{1}} \\
& \leq \sup _{\phi, \psi \in U} \mathbb{E}\|y(t, \phi)-y(t, \psi)\|_{\mathbb{H}}+\sup _{\phi, \psi \in U} \int_{-r}^{0} \mathbb{E}\|y(t+\theta, \phi)-y(t+\theta, \psi)\|_{\mathbb{H}} d \theta \\
& \leq \sup _{\phi, \psi \in U} M\left(1+r\|S\|_{\mathscr{L}\left(L_{r}^{2}\right)}\right)\left(1+e^{\gamma r}\right) e^{-\gamma t} \\
& \rightarrow 0 \quad \text { as } t \rightarrow \infty .
\end{array}
$$


Hence, there exists a stationary solution $\mu(\cdot)$ of $Y(t, \phi)$ for (6.12). On the other hand, if $\tilde{\mu}(\cdot) \in \mathscr{P}\left(\mathcal{H}_{1}\right)$ is also a stationary solution, then for any $f \in C_{L B}\left(\mathcal{H}_{1}\right)$, by the invariance of $\mu(\cdot), \tilde{\mu}(\cdot) \in \mathscr{P}\left(\mathcal{H}_{1}\right)$, it follows from (6.19) that

$$
|\mu(f)-\tilde{\mu}(f)| \leq \int_{\mathcal{H}_{1}} \int_{\mathcal{H}_{1}}\left|\mathbb{P}_{t} f(\phi)-\mathbb{P}_{t} f(\psi)\right| \mu(d \phi) \tilde{\mu}(d \psi) \leq C e^{-\gamma t}, \quad t \geq 0,
$$

for some $C>0$. This implies the uniqueness of stationary distributions by taking $t \rightarrow \infty$ in (6.20). The proof is complete now.

Examining the proof of Theorem 6.2, the technique employed therein applies to (6.12) with the Lévy triple $(0,0, \nu)$ and a retarded SDE (6.11) with an uniformly bounded diffusion coefficient $R$, i.e., $\sup _{x \in H}\|R(x)\|_{\mathscr{L}(K, H)}<\infty$.

\section{Example}

In this section, we shall consider an example to illustrate our theory in the previous sections. To this end, we assume that there exist $\eta:[-r, 0] \rightarrow \mathscr{L}(V, H)$ and $\eta:[-r, 0] \rightarrow$ $\mathscr{L}(H)$ of bounded variation such that

$$
M(\varphi)=\int_{-r}^{0} d \eta(\theta) \varphi(\theta) \quad \forall \varphi \in W^{1,2}([-r, 0], V),
$$

and

$$
N(\varphi)=\int_{-r}^{0} d \zeta(\theta) \varphi(\theta) \quad \forall \varphi \in W^{1,2}([-r, 0], H)
$$

Defining $\varrho: W^{1,2}([-r, 0], V) \times W^{1,2}([-r, 0], H) \rightarrow \mathbb{H}$ by $\varrho:=\left(\begin{array}{ll}0 & 0 \\ \eta & \zeta\end{array}\right)$, then we obtain that $F$ is of the form

$$
F\left(\begin{array}{c}
\varphi_{1} \\
\varphi_{1}
\end{array}\right)=\int_{-r}^{0} d \varrho(\theta)\left(\begin{array}{l}
\varphi_{1}(\theta) \\
\varphi_{1}(\theta)
\end{array}\right)
$$

Moreover, we can estimate for $a<0$,

$$
\left\|F_{a+i b} y\right\| \leq\left\|\int_{-r}^{0} d \varrho(\theta) e^{(a+i b) \theta} y\right\| \leq \operatorname{Var}(\varrho)_{-r}^{0} e^{-a r}\|y\| \quad \forall y \in \mathbb{H} .
$$

For simplicity, let $r=1$ in the sequel. Hence, Corollary 3.3 and Corollary 4.1 yield the following result.

Corollary 7.1. Under the assumptions of Corollary 4.1, the energy $\|y(t)\|_{\mathbb{H}}^{2}=\|u(t)\|_{V}^{2}+$ $\left\|u^{\prime}(t)\right\|_{H}^{2}, t \geq 0$, of the second-order abstract Cauchy problem 4.1) with delay decays exponentially if

$$
\sup _{b \in \mathbb{R}}\left\{\left\|\eta_{i b}\right\|_{\mathscr{L}(V, H)}+\left\|\zeta_{i b}\right\|_{\mathscr{L}(H)}\right\} \leq \frac{\alpha \kappa}{2 \alpha(3+\gamma)+\kappa}
$$

where $\alpha, \gamma$ and $\kappa$ are given as in Lemma 3.1 and Corollary 4.1 . 
Now let us consider a stochastic damped wave equation with delay

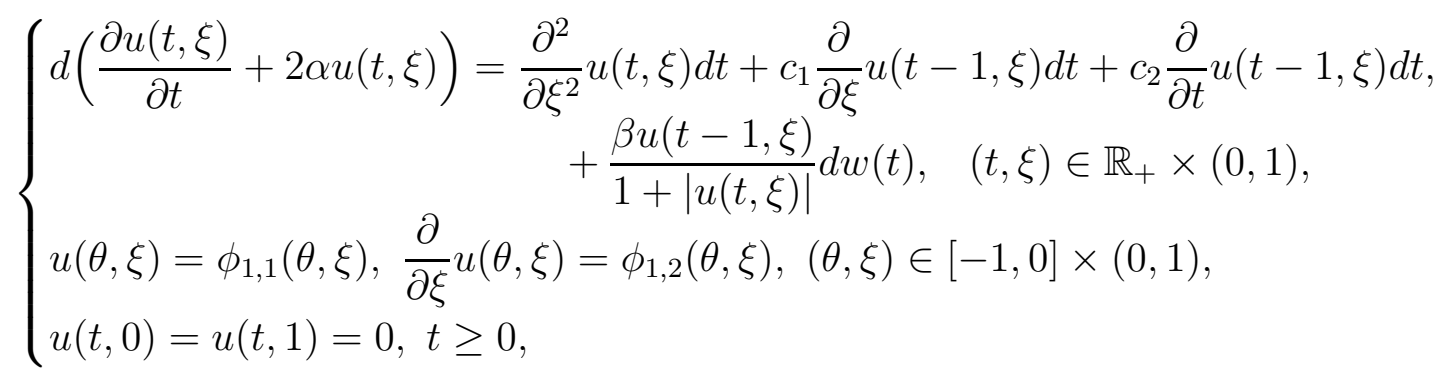

where $\alpha>0, \beta, c_{1}, c_{2} \in \mathbb{R}, c_{1} \neq 0$ or $c_{2} \neq 0$ and $w$ is a standard one-dimensional Brownian motion. Here we assume that $\phi_{1,1}(0, \cdot) \in H_{0}^{1}(0,1)$ with the mapping $\theta \rightarrow \phi_{1,1}(\theta, \cdot) \in H_{0}^{1}(0,1)$ belongs to $L^{2}\left([-1,0], H_{0}^{1}(0,1)\right)$, and $\phi_{1,2}(0, \cdot) \in L^{2}(0,1)$ with the mapping $\theta \rightarrow \phi_{1,2}(\theta, \cdot) \in$ $L^{2}(0,1)$ belongs to $L^{2}\left([-1,0], L^{2}(0,1)\right)$.

To write this problem in an abstract form, consider the space $V=H_{0}^{1}(0,1), H=L^{2}(0,1)$ and $\mathbb{H}:=V \times H$. We define the operators

$$
\begin{aligned}
& A=-\Delta, \mathscr{D}(A):=\left\{u \in H_{0}^{1}: \Delta u \in L^{2}(0,1)\right\} \\
& A^{1 / 2}=\sqrt{-\Delta}, \mathscr{D}\left(A^{1 / 2}\right)=H_{0}^{1}(0,1) \\
& B u=2 \alpha u, \mathscr{D}(B)=H \\
& \eta=c_{1} \frac{\partial}{\partial \xi} \delta_{-1}, \quad \zeta=c_{2} I \delta_{-1}
\end{aligned}
$$

where $\delta_{-1}$ is the point evaluation in -1 . We note that $\left\|A^{1 / 2} u\right\|_{H}=\||\nabla u|\|_{H}$ for $u \in V$ and $\left\{u \in H_{0}^{1}(0,1): \Delta u \in H\right\}=H_{0}^{2}(0,1)$.

Now let $\beta=0$ and by a direct calculation, it is not difficult to see that

$$
\sup _{b \in \mathbb{R}}\left\|\eta_{i b}\right\|_{\mathscr{L}(V, H)} \leq\left|c_{1}\right| \quad \text { and } \quad \sup _{b \in \mathbb{R}}\left\|\zeta_{i b}\right\|_{\mathscr{L}(H)} \leq\left|c_{2}\right|
$$

In Corollary 3.2, where we can consider a damped wave equation without delay, we choose

$$
\left(\left\|A^{1 / 2} B A^{-1 / 2}\right\|+2\left\|A^{-1 / 2}\right\|\right)^{-1}=\frac{\pi}{4 \alpha+2} .
$$

Then we have that the solution in this case decays exponentially if

$$
\left|c_{1}\right|+\left|c_{2}\right|<\frac{\alpha \pi}{36 \alpha+\pi}
$$

Now let us consider the stochastic delay partial differential equation (7.2) with $\beta \neq 0$. Define

$$
\gamma=\ln \frac{\alpha \pi}{\left(\left|c_{1}\right|+\left|c_{2}\right|\right)(36 \alpha+\pi)}>0
$$

then we have by virtue of (7.1) and Theorem 5.1 that whenever

$$
0<|\beta|<\frac{2}{3} \gamma e^{-2 \gamma}
$$


stochastic system (7.2) has a unique stationary process.

\section{References}

\section{References}

[1] Bao, J. H., Yin, G. and Yuan, C. G. Stationary distributions for retarded stochastic differential equations without dissipativity. Stochastics. 89, (2017), 530-549.

[2] Bátkai, A. and Piazzera, S. Semigroups for Delay Equations. Research Notes in Math., A.K. Peters, Wellesley, Massachusetts, (2005).

[3] Di Blasio, G., Kunisch, K. and Sinestrari, E. $L^{2}$-regularity for parabolic partial integrodifferential equations with delay in the highest-order derivatives. J. Math. Anal. Appl. 102 (1984), 38-57.

[4] Dudley, R. M. Real Analysis and Probability. Second Edition, Cambridge University Press, (2003).

[5] Engel, K-J. and Nagel, R. One-Parameter Semigroups for Linear Evolution Equations. Graduate Texts in Math. 194, New York, Springer-Verlag, (2000).

[6] Fattorini, H. Second Order Linear Differential Equations in Banach Spaces. North Holland Math. Studies Series, 108, (1985).

[7] Liang, F. and Gao, H. J. Stochastic nonlinear wave equation with memory driven by compensated Poisson random measures. J. Math. Phys. 55 (2014), 033503: 1-23.

[8] Liang, F. and Guo, Z. H. Asymptotic behavior for second order stochastic evolution equations with memory. J. Math. Anal. Appl. 419 (2014), 1333-1350.

[9] Liu, K. Stability of Infinite Dimensional Stochastic Differential Equations with Applications. Chapman \& Hall/CRC, London, New York, (2006).

[10] Liu, K. Stochastic retarded evolution equations: Green operators, convolutions and solutions. Stoch. Anal. Appl. 26 (2008), 624-650.

[11] Liu, K. A criterion for stationary solutions of retarded linear equations with additive noise. Stoch. Anal. Appl. 29 (2011), 799-823.

[12] Nakagiri, S. Structural properties of functional differential equations in Banach spaces. Osaka J. Math. 25, (1988), 353-398.

[13] Parthasarathy, K. P. Probability Measures on Metric Spaces. Academic Press, New York, (1967). 
[14] Ren, Y. and Sun, D. D. Second-order neutral impulsive stochastic evolution equations with delay. J. Math. Phys. 50 (2009), 102709: 1-12.

[15] Ren, Y. and Sakthivel, R. Existence, uniqueness and stability of mild solutions for second-order neutral stochastic evolution equations with infinite delay and Poisson jumps. J. Math. Phys. 53 (2012), 073517: 1-14.

[16] Taylor, A. and Lay, D. Introduction to Functional Analysis. Second Edition. John Wiley \& Sons, (1980). 